\title{
Density Forecast Evaluation in Unstable Environments
}

\author{
Gloria González-Rivera \\ Yingying Sun \\ Department of Economics \\ University of California, Riverside \\ and \\ Huazhong University of Science and Technology
}

August 2014 


\begin{abstract}
We propose a density forecast evaluation method in the presence of instabilities, which are defined as breaks in any conditional moment of interest and/or in the functional form of the conditional density of the process. We extend the battery of autocontour-based tests proposed in GonzálezRivera et al. $(2011,2014)$ by constructing Sup- and Ave-type tests calculated over a collection of subsamples in the evaluation period. These tests enjoy asymptotic distributions that are nuisanceparameter free, they are correctly sized and very powerful on detecting breaks in the parameters of the conditional mean and conditional variance. We also provide an accurate procedure to detect the location of the breaks and the unstable periods. We analyze the stability of a dynamic Phillips curve and find that the best one-step-ahead density forecast of changes in inflation is generated by a Markov switching model where the unemployment coefficient is state-dependent.
\end{abstract}

Key Words: Autocontour testing, Structural breaks, Phillips Curve

JEL Classification: C01, C22, C53. 


\section{Introduction}

Generally, instability is understood as changes in the parameters of a proposed forecasting model over the forecasting horizon. For clarification purposes, consider a simple model $y_{t+1}=\beta^{\prime} x_{t}+\sigma \varepsilon_{t+1}$ with $\varepsilon_{t} \sim$ i.i.d.N $(0,1)$. The model is unstable over time if the slope coefficients $\beta$ can change over the forecasting sample, either smoothly or abruptly to contain one or multiple breaks. We may also entertain a time varying variance such that $\sigma$ may be also subject to breaks, and we may have different conditional probability density functions, e.g. more or less thick tails, over different periods of time. This definition is general enough to account for most types of instability discussed in the current applied econometric literature. Up to today, the most comprehensive survey in the subject is Rossi (2014) in the Handbook of Economic Forecasting that reports extensive empirical evidence of instabilities in macroeconomic and financial data. Some examples follow.

The instability of predictive regressions, in which the significance of predictive regressors varies over different subsamples, has been documented in studies of predictability of stock returns (see Goyal and Welch, 2003; Paye and Timmermann, 2006; Rapach and Zhou, 2014), in exchange rate predictions (see Rossi, 2006; Rogoff and Stavrakeva, 2008) and in output growth and inflation forecasts (see Stock and Watson, 2003; Rossi and Sekhposyan, 2010). Naturally linked to this evidence is the econometric question on testing for parameter stability and structural breaks in the data, which has an illustrious history. From Chow (1960) test to most recent works such as Andrews (1993), Andrews and Ploberger (1994), Pesaran and Timmermann (2002), among others, testing for breaks has mainly focused on the behavior of the conditional mean. Our contribution aims to extend testing for instabilities to the full conditional density forecast that includes not only any conditional measure of interest, e.g. mean, variance, duration, etc. but also the functional form of the assumed conditional density function. Our analysis is framed more closely within the approach of Giacomini and Rossi (2009, 2010) and Rossi and Sekhposyan (2011) on the evaluation of out-of-sample forecasts in the presence of instability. However, differently from these works, we do not choose a particular loss function as we do not deal just with a point forecast but with the full density model. We ask: is the density forecast stable over time? and if it is not, where are the 
breaks? and to some extent, where are they coming from?

The testing methodology that we propose is based on the AutoContouR (ACR) device introduced by González-Rivera et al. (2011, 2012) and generalized later on in González-Rivera and Sun (2014). The null hypothesis of our tests is a correctly specified density forecast (joint hypothesis of correct dynamics in the moments of interest and correct functional form of the density). Following Diebold's (1998) seminal work, we work with Rosenblatt's probability integral transforms (PIT) associated with the point forecasts. Under the null, the PITs must be i.i.d uniformly distributed $\mathrm{U}[0,1]$. The Generalized AutoContouR (G-ACR) is a device (set of points) that is very sensitive to departures from the null in either direction and consequently, it provides the basis for very powerful tests. More specifically, for a time series of PITs, the G-ACRs are squares (in the univariate case) or hyper-cubes (in the multivariate case) of different (probability) areas or volumes within the maximum square or hyper-cube formed by a multidimensional uniform density $[0,1]^{n}$ for $n \geq 2$. By statistically comparing the location of the empirical PITs and the volume of the empirical G-ACRs with the location and volume of the population G-ACRs, we are able to construct a variety of tests for correct density forecast. Since the shapes of the G-ACRs can be visualized, we can extract information about where and how the rejection of the null hypothesis comes from. A great advantage of our approach is that it can also be applied quite easily to multivariate random processes of any dimension.

In a potential unstable data environment, we will form rolling subsamples within the forecasting sample. For every subsample, we apply a battery of G-ACR tests, and to detect instabilities, we construct a Sup- and an Ave-type statistics. Though the limiting distribution of these tests is a function of Brownian motions, the tests are nuisance-parameter free and their distribution can be tabulated. If the null hypothesis is rejected, we will be able to detect the timing of the break(s) rather accurately and the unstable periods. Though precise break detection is difficult, it is very important (Elliott and Muller, 2007) because it will help to improve the model forecast. Decisions regarding the choice of the estimation and evaluation windows and the estimation methods (e.g. recursive/rolling or time-varying parameter estimation) will be affected by the location of the break (Pesaran and Timmermann, 2007). In some instances, the break process can be modeled (Pesaran, 
Pettenuzzo, and Timmermann, 2006) and added to the model forecast.

The paper is organized as follows. In section 2, we review the G-ACR approach and introduce the new statistics with their asymptotic distributions. In section 3, we assess the finite sample properties (size and power) of the tests, and show how to detect potential breaks. We offer an extensive assessment by considering fixed, rolling, and recursive estimation schemes. In section 4, we apply the tests to assess the stability of the Phillips curve from 1958 on by evaluating the models proposed in Amisano and Giacomini (2007). We conclude in section 5. The appendix contains mathematical proofs.

\section{Statistics and Asymptotic Distributions}

\subsection{Construction of the Statistics}

The test statistics are based on the autocontour (ACR) and generalized autocontour (G-ACR) methodology proposed by González-Rivera et al. $(2011,2012,2014)$ that provides powerful tests for dynamic specification of the conditional density model either in-sample or out-of-sample environments. In the present context, we adapt these tests to instances where instabilities may be present in the data so that, beyond the evaluation of the density model, we will also be able to detect unstable periods.

Let $Y_{t}$ denote the random process of interest with conditional density function $f\left(y_{t} \mid \Omega_{t-1}\right)$, where $\Omega_{t-1}$ is the information set available up to time $t-1$. If the proposed predictive density model for $Y_{t}$, i.e. $\left\{f_{t}^{*}\left(y_{t} \mid \Omega_{t-1}\right)\right\}_{t=1}^{T}$ coincides with the true conditional density $\left\{f_{t}\left(y_{t} \mid \Omega_{t-1}\right)\right\}_{t=1}^{T}$, then the sequence of probability integral transforms (PIT) of $\left\{Y_{t}\right\}_{t=1}^{T}$ w.r.t $\left\{f_{t}^{*}\left(y_{t} \mid \Omega_{t-1}\right)\right\}_{t=1}^{T}$ i.e. $\left\{u_{t}\right\}_{t=1}^{T}$ must be i.i.d $U(0,1)$ where $u_{t}=\int_{-\infty}^{y_{t}} f_{t}^{*}\left(v_{t} \mid \Omega_{t-1}\right) d v_{t}$. Thus, the null hypothesis $H_{0}: f_{t}^{*}\left(y_{t} \mid \Omega_{t-1}\right)=f_{t}\left(y_{t} \mid \Omega_{t-1}\right)$ is equivalent to the null hypothesis $H_{0}^{\prime}:\left\{u_{t}\right\}_{t=1}^{T}$ is i.i.d $U(0,1)$ (see Diebold et al., 1998). Following González-Rivera and Sun (2014), we construct the generalized autocontours (G-ACR) under i.i.d. uniformity of predictive densities. Under $H_{0}^{\prime}:\left\{u_{t}\right\}_{t=1}^{T}$ i.i.d $U(0,1)$, the G-ACR $\alpha_{i}, k$ is defined as the set of points in the plane $\left(u_{t}, u_{t-k}\right)$ such that the square with $\sqrt{\alpha_{i}}$-side contains $\alpha_{i} \%$ of observations, 
i.e.,

$$
\text { G-ACR } \alpha_{\alpha_{i}, k}=\left\{B\left(u_{t}, u_{t-k}\right) \subset \Re^{2} \| 0 \leq u_{t} \leq \sqrt{\alpha_{i}} \text { and } 0 \leq u_{t-k} \leq \sqrt{\alpha_{i}} \text {, s.t. : } u_{t} \times u_{t-k} \leq \alpha_{i}\right\}
$$

We construct an indicator series $I_{t}^{k, \alpha_{i}}$ as follows

$$
I_{t}^{k, \alpha_{i}}=\mathbf{1}\left(\left(u_{t}, u_{t-k}\right) \subset \mathrm{G}-\mathrm{ACR}_{\alpha_{i}, k}\right)=\mathbf{1}\left(0 \leq u_{t} \leq \sqrt{\alpha_{i}}, 0 \leq u_{t-k} \leq \sqrt{\alpha_{i}}\right)
$$

Based on this indicator, González-Rivera and Sun (2014) proposed the following t-tests and chisquare statistics to test the null hypothesis $H_{0}^{\prime}:\left\{u_{t}\right\}_{t=1}^{T}$ i.i.d $U(0,1)$.

(1) t-ratio testing

$$
\frac{\sqrt{T-k}\left(\widehat{\alpha}_{i}-\alpha_{i}\right)}{\sigma_{k, i}} \rightarrow N(0,1)
$$

where $\widehat{\alpha_{i}}=\frac{\sum_{t=k+1}^{T} I_{t}^{k, \alpha_{i}}}{T-k}$, and $\sigma_{k, i}^{2}$ is the asymptotic variance of $\widehat{\alpha_{i}}$.

(2) chi-squared testing

(2.1) For a fixed autocontour $\alpha_{i}, L_{\alpha_{i}}^{\prime} \Lambda_{\alpha_{i}}^{-1} L_{\alpha_{i}} \rightarrow \chi_{K}^{2}$ where $L_{\alpha_{i}}=\left(\ell_{1, \alpha_{i}}, \ldots \ell_{K, \alpha_{i}}\right)^{\prime}$ is a $K \times 1$ stacked vector with element $\ell_{k, \alpha_{i}}=\sqrt{T-k}\left(\widehat{\alpha}_{i}-\alpha_{i}\right)$, and $\Lambda_{\alpha_{i}}$ is the asymptotic variance-covariance matrix of the vector $L_{\alpha_{i}}$.

(2.2) For a fixed lag $k, \mathbf{C}_{k}^{\prime} \Omega_{k}^{-1} \mathbf{C}_{k} \rightarrow \chi_{C}^{2}$ where $\mathbf{C}_{k}=\left(c_{k, 1}, \ldots c_{k, C}\right)^{\prime}$ is a $C \times 1$ stacked vector with element $c_{k, i}=\sqrt{T-k}\left(\widehat{\alpha_{i}}-\alpha_{i}\right)$, and $\Omega_{k}$ the asymptotic variance-covariance matrix of the vector $\mathrm{C}_{k}$.

In a potential unstable environment, we will construct the tests within the following rolling sample scheme. The total sample size $T$ is divided into two parts: in-sample observations (R) and out-ofsample observations (P). We form subsamples of size $r$ from $t-r+1$ up to $t$, where $t=R+r, \cdots, T$. In each subsample, we evaluate the proposed predictive density by calculating three different statistics $(t, C$ and $L)$. As a result, we obtain three sets of $n \equiv T-r-R+1$ tests each i.e., $\left\{t_{j}\right\}_{j=1}^{n},\left\{C_{j}\right\}_{j=1}^{n}$ and $\left\{L_{j}\right\}_{j=1}^{n}$. Finally, to detect instabilities, we construct Sup-type and Avg-type 
statistics by taking the supremum $(S)$ and the average $(A)$ respectively over each set $\left\{\left|t_{j}\right|\right\}_{j=1}^{n}$, $\left\{C_{j}\right\}_{j=1}^{n}$ and $\left\{L_{j}\right\}_{j=1}^{n}$ so that we obtain the following six statistics: $S_{|t|}, S_{C}, S_{L}$ and $A_{|t|}, A_{C}, A_{L}$.

\subsection{Asymptotic Properties of the Statistics}

Under the following set of assumptions, we provide three propositions, which proofs are provided in the Appendix.

- A1: For $T \rightarrow \infty, R \rightarrow \infty, P \rightarrow \infty, \lim _{T \rightarrow \infty} \frac{P}{R}=0$ and $\lim _{T \rightarrow \infty} \frac{r-k}{P}=m$, as $r, P \rightarrow \infty$, where $r$ is the size of the rolling subsample in the evaluation set, $m \in(0,1)$ and $k$ is the lag in the indicator $I_{t}^{k, \alpha_{i}}$.

- A2: $E\left|I_{t}^{k, \alpha_{i}}\right|^{q}<\Delta<\infty$ for some $q \geq 2$. This assumption is trivial as the second moments of the indicator are well defined as we will see next.

- A3: The data $\left\{y_{t}\right\}$ comes from a stationary and ergodic process ${ }^{1}$

Proposition 1 Let $J$ be the index that identifies a particular subsample in the evaluation period, i.e. $J=[P s], s \in[m, 1],[P m]=r-k$, and let $\widehat{\alpha}_{i}(J)=\frac{\sum_{t=R+1+J-r+k}^{R+J} I_{t}^{k, \alpha_{i}}}{r-k}$ be the corresponding subsample proportion based on the indicator. The Sup- and Avg-tests are

$$
\begin{aligned}
& S_{|t|}=\sup _{J}\left|\frac{\sqrt{r-k}\left(\widehat{\alpha}_{i}(J)-\alpha_{i}\right)}{\sigma_{k, \alpha_{i}}}\right| \\
& A_{|t|}=\frac{1}{P-r+1} \sum_{\underline{J}}\left|\frac{\sqrt{r-k}\left(\widehat{\alpha}_{i}(J)-\alpha_{i}\right)}{\sigma_{k, \alpha_{i}}}\right|
\end{aligned}
$$

where $\sigma_{k, \alpha_{i}}^{2}=\alpha_{i}\left(1-\alpha_{i}\right)+2 \alpha_{i}^{3 / 2}\left(1-\alpha_{i}^{1 / 2}\right)$

\footnotetext{
${ }^{1}$ This assumption can be relaxed to include more general mixing processes because the relevant conditions to invoke limiting theorems as the FCLT are those of the indicator process.
} 
Given assumptions A1-A3, and under the null hypothesis of i.i.d $U(0,1)$ PITs, the asymptotic distribution of the tests are as follows,

$$
\begin{aligned}
& S_{|t|} \underset{P \rightarrow \infty}{\longrightarrow} \sup _{s \in[m, 1]} \frac{|W(s)-W(s-m)|}{\sqrt{m}} \\
& A_{|t|} \underset{P \rightarrow \infty}{\longrightarrow} \int_{\underline{s}}^{\bar{s}} \frac{|W(s)-W(s-m)|}{\sqrt{m}} d s
\end{aligned}
$$

where $W($.$) is a standard univariate Brownian motion.$

Proposition 2 For a given lag $k$, write $c_{k, i}(J)=\sqrt{r-k}\left(\widehat{\alpha_{i}}(J)-\alpha_{i}\right)$ and stack $c_{k, i}(J)$ for different autocontours levels $i=1,2, \ldots C$ such that $\mathbf{C}_{k}(J)=\left(c_{k, 1}(J), \ldots c_{k, C}(J)\right)^{\prime}$ is the $C \times 1$ stacked vector. The Sup- and Avg-tests are

$$
\begin{aligned}
& S_{C}=\sup _{J} \mathbf{C}_{k}(J)^{\prime} \Omega_{k}^{-1} \mathbf{C}_{k}(J) \\
& A_{C}=\frac{1}{P-r+1} \sum_{\underline{J}} \mathbf{C}_{k}(J)^{\prime} \Omega_{k}^{-1} \mathbf{C}_{k}(J)
\end{aligned}
$$

where $\Omega_{k}$ is the asymptotic variance and covariance matrix of the random vector $\mathbf{C}_{k}(J)$. Given assumptions A1-A3, and under the null hypothesis of $i . i . d U(0,1)$ PITs, the asymptotic distribution of the tests are as follows,

$$
\begin{aligned}
& S_{C} \underset{P \rightarrow \infty}{\longrightarrow} \sup _{s \in[m, 1]} \frac{(\mathbf{W}(s)-\mathbf{W}(s-m))^{\prime}(\mathbf{W}(s)-\mathbf{W}(s-m))}{m} \\
& A_{C} \underset{P \rightarrow \infty}{\longrightarrow} \int_{\underline{s}}^{\bar{s}} \frac{(\mathbf{W}(s)-\mathbf{W}(s-m))^{\prime}(\mathbf{W}(s)-\mathbf{W}(s-m))}{m} d s
\end{aligned}
$$

where $\mathbf{W}($.$) is a standard C$-variate Brownian motion.

Proposition 3 For a given contour $\alpha_{i}$, write $\ell_{k, \alpha_{i}}(J)=\sqrt{r-k}\left(\widehat{\alpha}_{i}(J)-\alpha_{i}\right)$ and stack $\ell_{k, \alpha_{i}}$ for $k=1, \ldots K$. Let $\mathbf{L}_{\alpha_{i}}(J)=\left(\ell_{1, \alpha_{i}}(J), \ldots \ell_{K, \alpha_{i}}(J)\right)^{\prime}$ be the $K \times 1$ stacked vector. The Sup- and 
Avg-tests are

$$
\begin{aligned}
S_{L} & =\sup _{J} \mathbf{L}_{\alpha_{i}}(J)^{\prime} \Lambda_{\alpha_{i}}^{-1} \mathbf{L}_{\alpha_{i}}(J) \\
A_{L} & =\frac{1}{P-r+1} \sum_{\underline{J}}\left|\mathbf{L}_{\alpha_{i}}(J)^{\prime} \Lambda_{\alpha_{i}}^{-1} \mathbf{L}_{\alpha_{i}}(J)\right|
\end{aligned}
$$

where $\Lambda_{\alpha_{i}}$ is the asymptotic variance and covariance matrix for the random vector $\mathbf{L}_{\alpha_{i}}$. Given assumptions A1-A3, and under the null hypothesis of $i . i . d U(0,1)$ PITs, the asymptotic distribution of the tests are as follows,

$$
\begin{aligned}
& S_{L} \underset{P \rightarrow \infty}{\longrightarrow} \sup _{s \in[m, 1]} \frac{(\mathbf{W}(s)-\mathbf{W}(s-m))^{\prime}(\mathbf{W}(s)-\mathbf{W}(s-m))}{m} \\
& A_{L} \underset{P \rightarrow \infty}{\longrightarrow} \int_{\underline{s}}^{\bar{s}} \frac{(\mathbf{W}(s)-\mathbf{W}(s-m))^{\prime}(\mathbf{W}(s)-\mathbf{W}(s-m))}{m} d s
\end{aligned}
$$

where $\mathbf{W}($.$) is a standard L$-variate Brownian motion.

We tabulate the percentiles of the asymptotic distributions of the tests provided in Propositions 1 to 3. Since the distributions depend on $m$, which is proportion of the rolling sample to the total evaluation sample, we consider the following values of $m \in[0.1,0.2,0.3,0.4,0.5,0.6,0.7,0.8,0.9]$. In Table 1. we report the percentiles of the distribution of the $S_{|t|}$ and $A_{|t|}$ statistics; in Table 2 , those for the $S_{C}$ and $A_{C}$ considering the 13 autocontour $C=[0.01,0.05,0.1,0.2,0.3,0.4,0.5,0.6,0.7,0.8,0.9,0.95,0.99]$; and in Table 3 those for the $S_{L}$ and $A_{L}$ tests considering 5 lags.

[TABLES 1-3 ABOUT HERE]

\section{Monte Carlos Simulations}

We perform extensive Monte Carlos simulations to assess the finite sample properties (size and power) of the proposed statistics. For $\sqrt{R}$-consistent estimators of the parameters of the model, i.e. $\left(\widehat{\theta}-\theta_{0}\right)=O_{p}\left(R^{-1 / 2}\right)$ with a well-defined asymptotic distribution, and under assumption A1, $R \rightarrow \infty, P \rightarrow \infty$, and $P / R \rightarrow 0$ as $T \rightarrow \infty$, parameter uncertainty is asymptotically 
negligible. In this case, the critical values tabulated in Tables 1 to 3 can be used directly. In cases where the condition $P / R \rightarrow 0$ is violated, we can either bootstrap the variance of the tests (and use the tabulated critical values) or the tests themselves to approximate their asymptotic distribution. In the following simulations, we keep the ratio $P / R$ constant and we implement a parametric bootstrap for the distribution of the statistics. This is a sensible approach because the null hypothesis fully specifies the parametric data generating process. We consider fixed, rolling, and recursive forecasting schemes.

\subsection{Size of the Tests}

Under the null hypothesis of a stable density model, we consider the following data generating process: $y_{t}=\alpha_{1}+\beta_{1} y_{t-1}+\beta_{2} x_{t-1}+\sigma \epsilon_{t}$ where $x_{t}=\phi_{1}+\phi_{2} x_{t-1}+\varepsilon_{t}$, and $\varepsilon_{t} \sim N(0,1), \phi_{1}=1.38$, $\phi_{2}=0.77, \alpha_{1}=1.5, \beta_{1}=0.5, \beta_{2}=0.6, \sigma=1$. We consider sample sizes of $T=150$ (with evaluation sample $P=60$ ), $T=375$ (with $P=150$ ), and $T=750$ (with $P=300$ ) observations, and for each sample size, we consider the proportion $m=r / P$ equal to $m=1 / 3, m=1 / 2$ and $m=2 / 3$. We maintain the ratio $P / R$ constant and equal to $2 / 3$. In total, we run nine experiments, of which we present here the two most extremes: small sample size with small subsample window $(T=150, m=1 / 3)$ and large sample size with large subsample window $(T=750, m=2 / 3)$. The size results of these two cases are presented in Tables 4 and 5 respectively. The remaining seven cases are available in a "supplementary material" file. We work with 13 autocontour coverage levels $C=[0.01,0.05,0.1,0.2,0.3,0.4,0.5,0.6,0.7,0.8,0.9,0.95,0.99]$. The number of Monte Carlo replications is 1,000, and the number of bootstrap samples is 500 .

\section{[TABLES 4-5 ABOUT HERE]}

The overall size of the tests is very good in the nine experiments considered. There are not substantial differences among the fixed, rolling, and recursive estimation schemes. We find some small size distortions (under-sized) when the sample is small $(T=150)$ and the autoncotour levels are extreme (1\% and $99 \%$ ) but as the sample size increases, the distortion disappears. For the individual tests $S_{|t|}^{k, \alpha_{i}}$ and $A_{|t|}^{k, \alpha_{i}}$ ( $k$ and $\alpha_{i}$ fixed), the Ave-test tends to have better size than the 
Sup-test. The umbrella tests $S_{C}, A_{C}, S_{L}$, and $A_{L}$ have very good size even in small samples.

\subsection{Power of the Tests}

To assess the power of the tests, we generate data from four different processes, all of them containing a break point. The model that we maintain under the null hypothesis is the same as the one considered in the study of the size properties: $y_{t}=\alpha_{1}+\beta_{1} y_{t-1}+\beta_{2} x_{t-1}+\sigma \epsilon_{t}$ with $x_{t}=\phi_{1}+\phi_{2} x_{t-1}+\varepsilon_{t}, \varepsilon_{t} \sim N(0,1)$. The total sample size $(T)$ is $650, R=350, P=300$, and $m=1 / 3$. The break point happens at at $R+\tau P$, where $\tau=1 / 3$. In the following experiments, the number of Monte Carlo replications is 1000 and bootstrapped samples 500. We maintain a nominal test size of $5 \%$.

The four data generating mechanisms are the following:

DGP1: Break in the intercept of $y_{t}=\alpha_{t}+\beta_{1} y_{t-1}+\beta_{2} x_{t-1}+\sigma \epsilon_{t}, \epsilon_{t} \sim N(0,1)$ :

$$
\alpha_{t}= \begin{cases}\alpha_{1}=1.5 & \text { if } t<\text { break } \\ \alpha_{2}=2 & \text { otherwise }\end{cases}
$$

with $\beta_{1}=0.5, \beta_{2}=0.6, \sigma=1$.

DGP2: Break in the variance of $y_{t}=\alpha+\beta_{1} y_{t-1}+\beta_{2} x_{t-1}+\sigma_{t} \epsilon_{t}, \epsilon_{t} \sim N(0,1)$ :

$$
\sigma_{t}= \begin{cases}\sigma_{1}=1.5 & \text { if } t<\text { break } \\ \sigma_{2}=1.8 & \text { otherwise }\end{cases}
$$

with $\alpha=1.5, \beta_{1}=0.5, \beta_{2}=0.6$.

DGP3: Breaks in the slope coefficients of $y_{t}=\alpha+\beta_{1, t} y_{t-1}+\beta_{2, t} x_{t-1}+\sigma \epsilon_{t}, \epsilon_{t} \sim N(0,1)$ :

$$
\beta_{1, t}= \begin{cases}\beta_{1,1}=0.5 & \text { if } t<\text { break } \\ \beta_{1,2}=0.3 & \text { otherwise }\end{cases}
$$




$$
\beta_{2, t}= \begin{cases}\beta_{2,1}=0.6 & \text { if } t<\text { break } \\ \beta_{2,2}=0.4 & \text { otherwise }\end{cases}
$$

with $\alpha=1.5, \sigma=1$.

DGP4: Breaks in the intercept, variance and slope coefficients of $y_{t}=\alpha_{t}+\beta_{1, t} y_{t-1}+\beta_{2, t} x_{t-1}+\sigma_{t} \epsilon_{t}$, $\epsilon_{t} \sim N(0,1)$ :

$$
\begin{gathered}
\alpha_{t}= \begin{cases}\alpha_{1}=1.5 & \text { if } t<\text { break } \\
\alpha_{2}=2 & \text { otherwise }\end{cases} \\
\sigma_{t}= \begin{cases}\sigma_{1}=1.5 & \text { if } t<\text { break } \\
\sigma_{2}=1.8 & \text { otherwise }\end{cases} \\
\beta_{1, t}= \begin{cases}\beta_{1,1}=0.5 & \text { if } t<\text { break } \\
\beta_{1,2}=0.3 & \text { otherwise }\end{cases} \\
\beta_{2, t}= \begin{cases}\beta_{2,1}=0.6 & \text { if } t<\text { break } \\
\beta_{2,2}=0.4 & \text { otherwise }\end{cases}
\end{gathered}
$$

Note that the breaks considered are not very extreme. We perform all the simulations under fixed, rolling, and recursive estimation schemes. We report the power results for the fixed and rolling schemes in Tables 6 to 9 , and those for the recursive scheme in the supplementary material.

\section{[TABLES 6-9 ABOUT HERE]}

Under the fixed scheme, the tests are most powerful (power of about 90\%) to detect breaks in intercept and slope coefficients (DGP1, DGP3, and DGP4). Both Ave- and Sup- tests enjoy similar performance, either for single hypothesis $\left(S_{|t|}\right.$ and $\left.A_{|t|}\right)$ or for joint hypothesis $\left(S_{C}, A_{C}, S_{L}\right.$, $A_{L}$ ). The power drops when testing for breaks in the variance (DGP2) with values of $10-60 \%$ for the single hypothesis tests and of $60 \%$ for the joint hypothesis tests. Under the rolling scheme, as we expected, the tests are less powerful overall because by rolling the estimation sample, the model adjusts slowly to the new parameters values. Nevertheless, the power of $S_{C}, A_{C}, S_{L}$ and $A_{L}$ is still very high (around 50-80\%) for DGP1, DGP3, and DGP4, and around 40\% for DGP2. For all DGPs, the Ave-test is more powerful that the Sup. Under the recursive scheme, the tests 
performance is slightly worse than in the fixed scheme but slightly better than in the rolling scheme.

\subsection{Detection of the break point}

An advantage of these tests is that they are very helpful to detect the location of the break in the data. In this section, we will show how to search for the break. We consider two cases: a break in the intercept of the model (DGP1B) and a break in the variance (DGP2B). We generate a time series of $T=600$ observations from each of the following processes:

DGP1B: $y_{t}=\alpha_{t}+\beta_{1} y_{t-1}+\beta_{2} x_{t-1}+\sigma \epsilon_{t}, \epsilon_{t} \sim N(0,1)$

$$
\alpha_{t}= \begin{cases}\alpha_{1}=1.5 & \text { if } t<\text { break } \\ \alpha_{2}=0.1 & \text { otherwise }\end{cases}
$$

with $\beta_{1}=0.5, \beta_{2}=0.6, \sigma=1$, and the break occuring at the 480 observation, i.e. break $=480$. DGP2B: $y_{t}=\alpha+\beta_{1} y_{t-1}+\beta_{2} x_{t-1}+\sigma_{t} \epsilon_{t}, \epsilon_{t} \sim N(0,1)$

$$
\sigma_{t}= \begin{cases}\sigma_{1}=2 & \text { if } t<\text { break } \\ \sigma_{2}=0.5 & \text { otherwise }\end{cases}
$$

with $\alpha=1.5, \beta_{1}=0.5, \beta_{2}=0.6$, and break=480 observation.

We proceed by estimating the model $y_{t}=\alpha+\beta_{1} y_{t-1}+\beta_{2} x_{t-1}+\sigma \epsilon_{t}, \epsilon_{t} \sim N(0,1)$ and choosing the following samples $\mathrm{R}=350, \mathrm{P}=250$, and $m=r / P=100 / 250=0.4$. We implement the three estimation schemes (fixed, recursive, and rolling). For each subsample $(r=100)$ in the evaluation window $P$ we calculate the t-ratio and $\mathrm{C}$ statistics for a total of 151 tests $(T-R+1-r)$. Next, from the asymptotic distribution tables, we choose the $5 \%$ critical values corresponding to $S_{|t|}$ and $S_{C}$ as the thresholds that will help to locate the first significant subsample that should contain the break point. Given the sample windows that we have chosen, i.e. $P / R=0.71$, we recommend bootstrapping the tests $S_{|t|}$ and $S_{C}$ in order to find the $5 \%$ critical value. In Figures 1 and 2 , we plot the 151 t-tests and C-tests in a sequential fashion together with the bootstrapped $5 \%$ critical 
values corresponding to the distribution of $S_{|t|}$ and $S_{C}$ under the three estimation schemes and for both DGP1B and DGP2B.

Regarding DGP1B, the C-tests react faster to the break than the t-tests for all estimation schemes. For instance, under the fixed scheme, it is the 80th t-statistics that hits the threshold for the first time (from that point on all t-tests are above the threshold) and it marks the first subsample that contains the break, which corresponds to the interval of observations [430, 529]. For the C-statistics, it is the 36th test hitting the threshold line for the first time, which corresponds to the observations in the subsample $[386,485]$. The break must be contained in the intersection of these two subsamples, i.e. [430, 485]. The actual break happens at the 480th observation. We observe that the maximum values of the t-tests and C-tests are those of the 130th statistic, which corresponds to the subsample $[480,579]$. From this point on, the values of the tests start decreasing, which means that the break point is already passed. As expected, under the recursive and rolling schemes, the tests react slower to the break but nevertheless they are also accurate on pointing out the subsample where the break occurs.

Regarding DGP2B, both t-tests and C-tests react at about the same time. For instance, under the recursive scheme, it is the 73th statistic (for both $\mathrm{t}$ and $\mathrm{C}$ tests) that hits the threshold for the first time. This corresponds to the subsample [423, 522]. The maximum values of the t-tests and C-tests are those of the 130th statistic, which corresponds to the subsample [480, 579], and putting these two pieces of information together, we locate the break within the observations [480, 522]. 

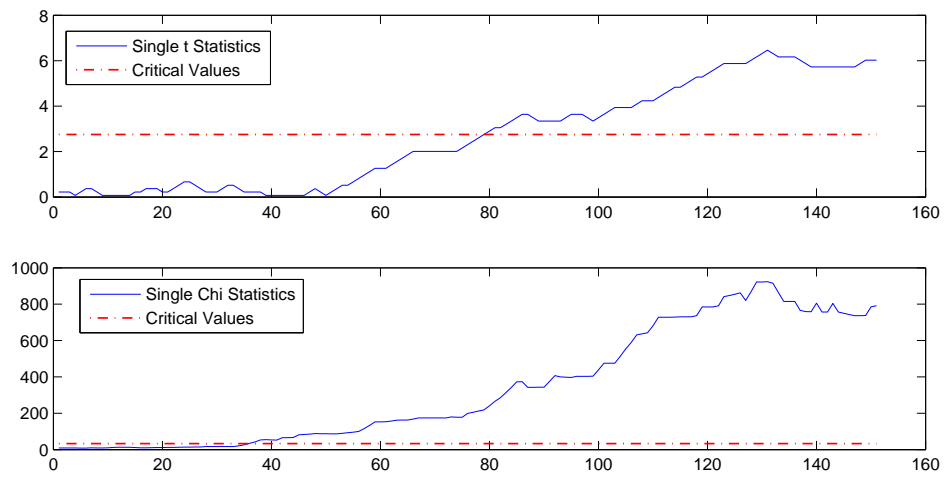

Fixed Scheme
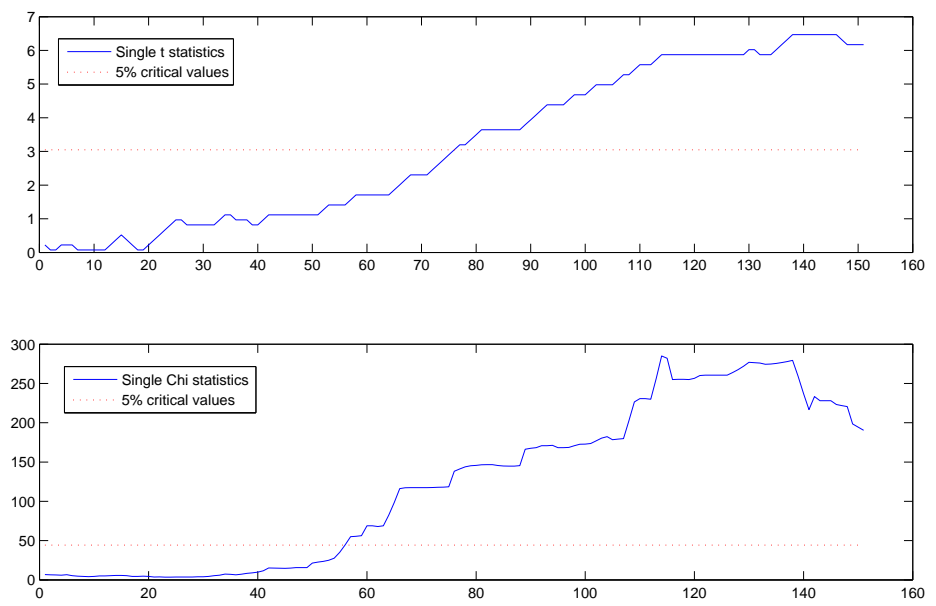

Recursive Scheme
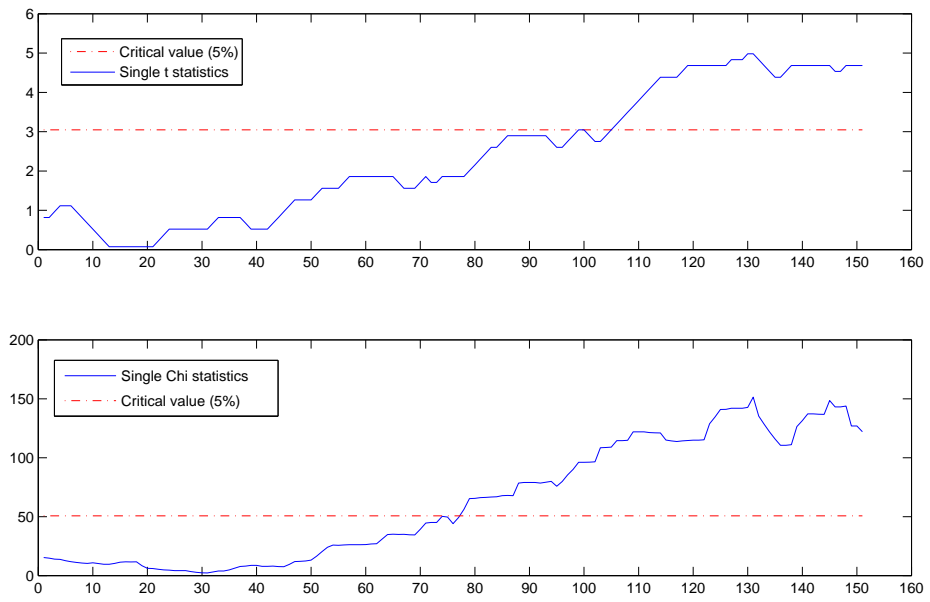

Rolling Scheme

Figure 1: Plots of $t$ and $C$ Statistics for DGP1B: Break in the intercept 

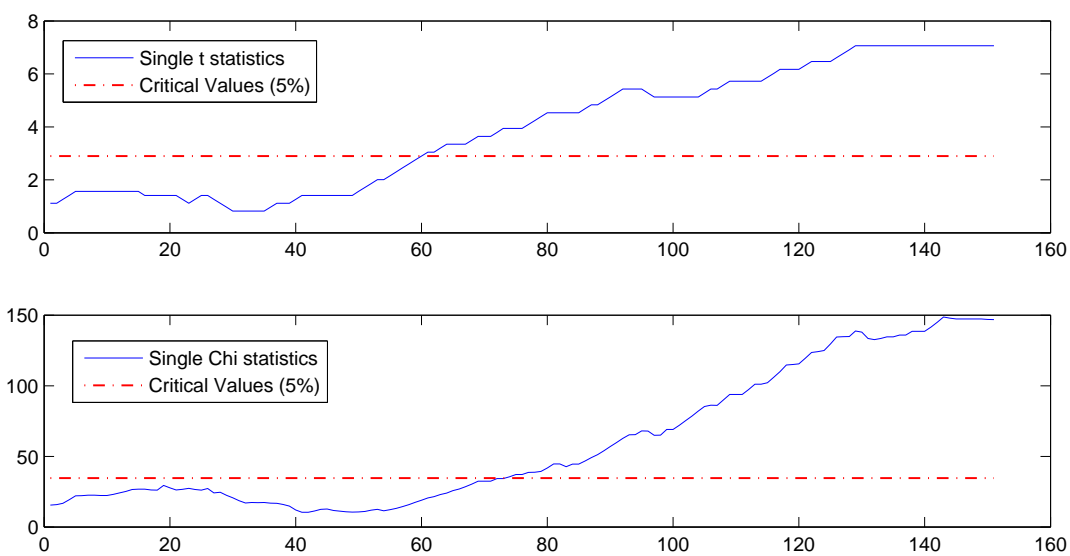

Fixed Scheme
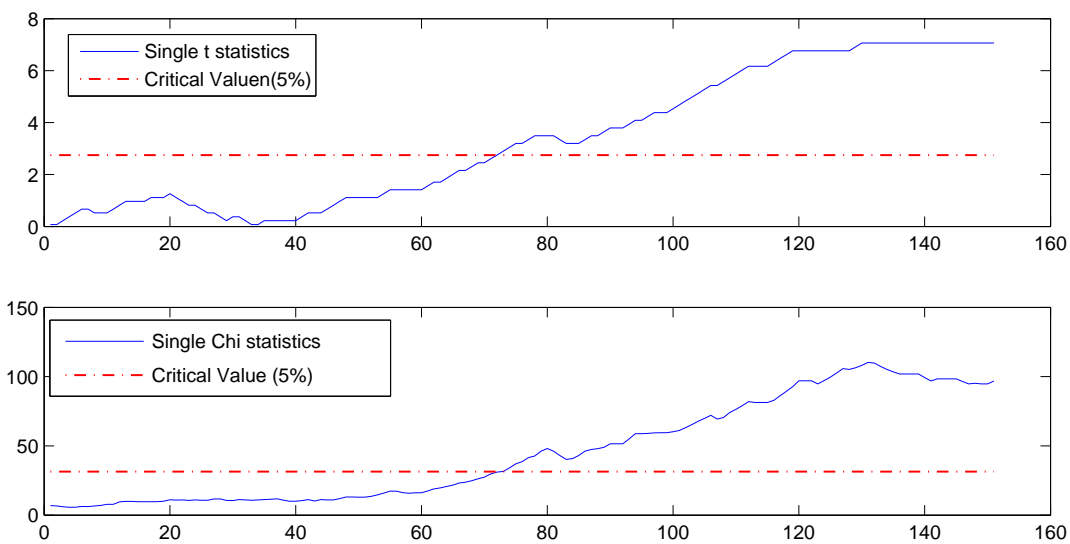

Recursive Scheme
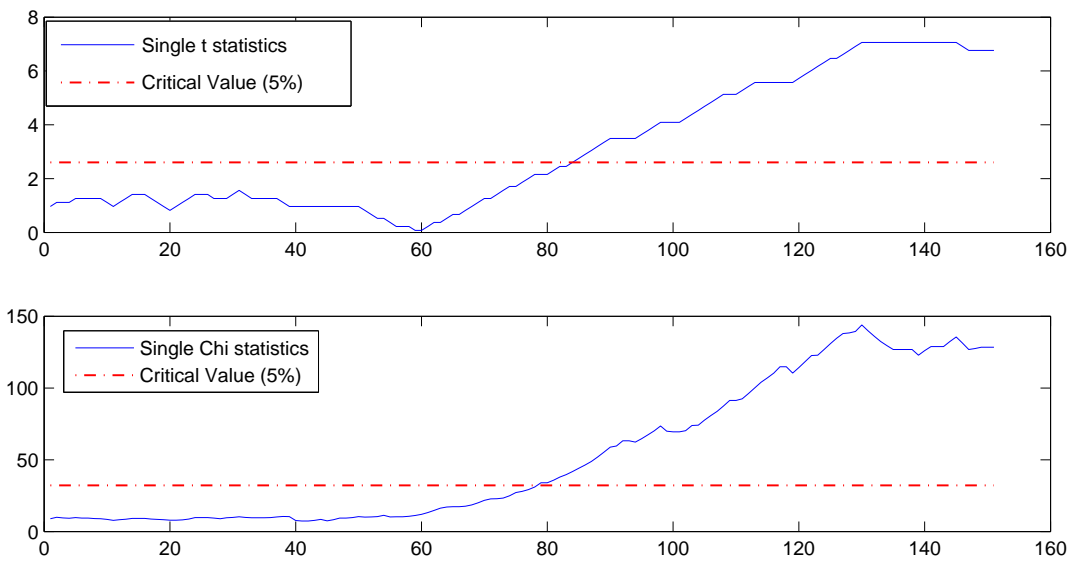

Rolling Scheme

Figure 2: Plots of $t$ and $C$ Statistics for DGP2B: Break in the variance 


\section{Density Forecast Evaluation of the Phillips Curve}

We apply the proposed tests to analyze the stability of the Phillips curve. Stock and Watson (1999) found some empirical evidence in favor of the Phillips curve as a forecasting tool, they showed that inflation forecasts produced by the Phillips curve were more accurate than forecasts based on simple autoregressive or multivariate models but they also found parameter instabilities across different subsamples. Rossi and Skehposyan (2010) showed that the predictive power of the Phillips curve disappeared around the time of the Great Moderation. Based on scoring rules, Amisano annd Giacomini (2007) compared the density forecast accuracy of several models of the Phillips curve and concluded that the best density forecast is produced by a Markov-switching model. Since their comparisons are based on the average forecasting performance of competing models over time, they cannot directly address the instabilities widely documented in the literature. In this section, we consider the models in Amisano annd Giacomini (2007) and we focus on their absolute density forecast performance in the presence of instabilities. Our starting model is a linear Phillips curve (Stock and Watson, 1999), in which changes of the inflation rate depend on their lags and on lags of the unemployment rate i.e.,

$$
\Delta \pi_{t}=\alpha_{1}+\beta_{1} \Delta \pi_{t-1}+\beta_{2} \Delta \pi_{t-2}+\beta_{12} \Delta \pi_{t-12}+\gamma u_{t-1}+\sigma \epsilon_{t}
$$

where $\pi_{t}=100 \times \ln \left(C P I_{t} / C P I_{t-12}\right) ; C P I_{t}$ is the consumer price index for all urban consumers and all items; $u_{t-1}$ is the civilian unemployment rate; and $\epsilon_{t} \sim N(0,1)$. The dats is collected from the FRED database; monthly CPI and unemployment series are both seasonally adjusted. The time series run from 1958M01 to 2012M01 (updated sample from 1958M01-2004M07 in Amisano and Giacomini). Standard tests on $\Delta \pi_{t}$ and $u_{t}$ show that they do not have a unit root. On implementing our tests, we consider the same estimation sample as in Amisano and Giacomini, from 1958M01 to 1987M12 (360 observations). The evaluation sample runs from $1988 \mathrm{M} 01$ to 2012M01 (289 observations) with subsamples of size $r=200$. 


\subsection{Evaluation of the Linear Phillips Curve}

We present the evaluation results for the linear Phillips Curve in Table 10 under fixed and rolling estimation schemes. The recursive case is in the supplementary material.

\section{[TABLE 10 ABOUT HERE]}

The umbrella tests $S_{C}, A_{C}, S_{L}$, and $A_{L}$ indicate a clear rejection of the linear model. On examining the individual tests $S_{|t|}$ and $A_{|t|}$, the rejection comes from the middle autocontours, between $40 \%$ and $70 \%$ coverage, and from the 95-99\% levels (large changes in inflation). In Figure 3 , we plot the t- and C-statistics over the evaluation period in a sequential fashion. The tests break through their corresponding critical values around the 60th statistic. From this point on, the values of the tests keep on increasing reaching two local maxima, which points to two potential breaks: the first in the 64th statistic for the t-tests (1993M03 to 2009M11) and in the 71th statistic for the C tests (1993M11 to 2010M06), and the second local maximum in the 78th statistic that corresponds to the period 1994M06 to 2011M01. All these periods include the years 1993-2007 in the so-called Great Moderation and the years after the deep financial crisis of 2008.

\subsection{Evaluation of Non-Linear Phillips Curve}

Given the rejection of the linear Phillips curve, we proceed with a flexible specification by assuming that the coefficients in the linear model vary according to a Markov switching mechanism. We consider a two-state Markov switching model, i.e.,

$$
\Delta \pi_{t}=\alpha^{s_{t}}+\beta_{1}^{s_{t}} \Delta \pi_{t-1}+\beta_{2}^{s_{t}} \Delta \pi_{t-2}+\beta_{12}^{s_{t}} \Delta \pi_{t-12}+\gamma^{s_{t}} u_{t-1}+\sigma^{s_{t}} \epsilon_{t}
$$

where the unobserved state variable $s_{t}$ switches between two states, 1 or 2 , with transition probabilities $\operatorname{Pr}\left(s_{t}=j \mid s_{t-1}=i\right)=p_{i j}$ for $i, j=1,2$; and $\epsilon_{t}$ is assumed to be a standard normal variate. Thus, this model allows for non-Gaussian density forecasts. Since all the coefficients depend on the state variable (Model 1), the model is extremely flexible and it will adapt to potential breaks or instabilities that may occur over time. We have run our test statistics and we fail to reject the null 

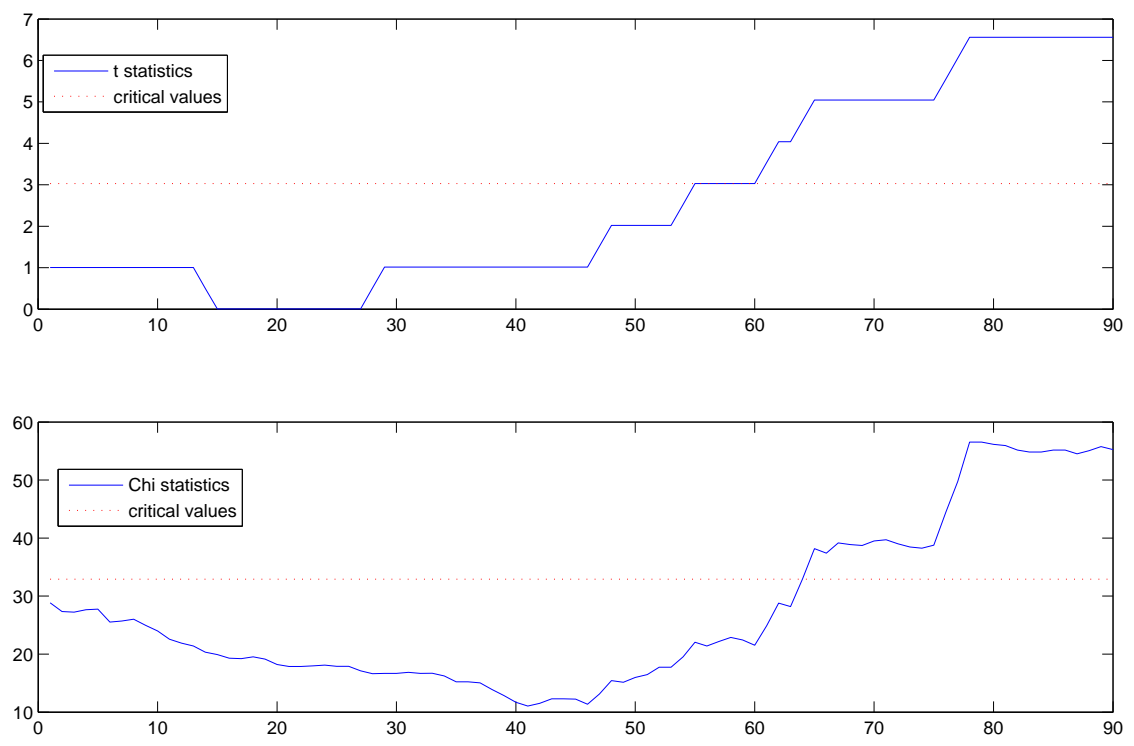

Figure 3: Plots of t-ratio (99\% autocontour) and $C$ Statistics for Linear Phillips Curve (fixed scheme)

hypothesis of correct specification. However, we would like to investigate what coefficients are key to understand where the nonlinear behavior comes from. We consider two additional specifications,

Model 2: $\Delta \pi_{t}=\alpha+\beta_{1}^{s t} \Delta \pi_{t-1}+\beta_{2}^{s t} \Delta \pi_{t-2}+\beta_{12}^{s t} \Delta \pi_{t-12}+\gamma u_{t-1}+\sigma \epsilon_{t}$, (intercept and unemployment coefficient do not depend on $s_{t}$ ).

Model 3: $\Delta \pi_{t}=\alpha^{s_{t}}+\beta_{1} \Delta \pi_{t-1}+\beta_{2} \Delta \pi_{t-2}+\beta_{12} \Delta \pi_{t-12}+\gamma^{s t} u_{t-1}+\sigma_{s_{t}} \epsilon_{t}$, (inflation coefficients do not depend on $\left.s_{t}\right)$.

We present the test results in Table 11 .

\section{[TABLE 11 ABOUT HERE]}

For Model 2, we still fail to reject the overall model as the umbrella tests $S_{C}, A_{C}, S_{L}$, and $A_{L}$ have p-values larger than 5\%. However, on a close examination of the individual tests $S_{|t|}$ and $A_{|t|}$, we observe a rejection of the model in the autocontours 90 to $99 \%$. In Model 3, we allow the intercept, the unemployment coefficient and the variance of the error to be state dependent while the rest of the parameters are constant. In this case, all statistics (joint and individual) 
enjoy p-values larger than $5 \%$ and consequently we fail to reject the model even in the presence of instabilities. A nonlinear Phillips curve has better predictive performance than a linear model, which is unstable over time. The advantage comes from letting the coefficient that links inflation and unemployment to be state-dependent.

\section{Conclusion}

We have provided a battery of tests to assess the stability of the density forecast over time, which offer important advantages for the empirical researcher. These tests are nuisance-parameter free and their asymptotic distributions can be tabulated. If the tests reject the null hypothesis of a stable density forecast, the shapes of the empirical generalized autocontours can be visualized to extract information regarding the direction of the rejection. Regardless of the estimation scheme (fixed, rolling, or recursive), their finite sample properties are superior. In some instances, the Ave-tests tend to have a slightly better size than the Sup-tests. Both types are more powerful on detecting breaks in the intercept and slope coefficients than on detecting breaks in the variance. In addition, we have proposed a rather accurate procedure to find the location of the breaks and the unstable periods. The tests can also be easily applied to multivariate random processes of any

dimension. As an application of the tests, we have analyzed the stability of the Phillips curve. A linear model is strongly rejected in favor of a non-linear specification that allows the coefficient linking inflation changes and unemployment to be state-dependent. The break of the linear model occurs during the Great Moderation years. 


\section{Appendix}

\section{Proof of Proposition 1:}

The indicator function $I_{t}^{k, \alpha_{i}}$ is a Bernoulli random variable with the following moments: $E\left(I_{t}^{k, \alpha_{i}}\right)=$ $\alpha_{i}, \operatorname{Var}\left(I_{t}^{k, \alpha_{i}}\right)=\alpha_{i}\left(1-\alpha_{i}\right)$ and covariance

$$
r_{h}^{\alpha_{i}} \equiv \operatorname{cov}\left(I_{t}^{k, \alpha_{i}}, I_{t-h}^{k, \alpha_{i}}\right)=\left\{\begin{array}{cc}
0 & \text { if } h \neq k \\
\alpha_{i}^{3 / 2}\left(1-\alpha_{i}^{1 / 2}\right) & \text { if } h=k
\end{array}\right.
$$

Since the indicator process is stationary and ergodic, $\widehat{\alpha_{i}}(J)$ satisfies the condition of global covariance stationarity required for the FLCT to apply (Theorem 7.17) in White (2001). Since $J=[P s]$, $s \in[m, 1]$, and $r-k=[P m]$, we write

$$
\begin{aligned}
W_{P}(s) & \equiv \frac{\sqrt{(r-k)}\left(\widehat{\alpha_{i}}(J)-\alpha_{i}\right)}{\sigma_{k, i}}=\frac{\sum_{t=R+1+J-r+k}^{R+J}\left(I_{t}^{k, \alpha_{i}}-\alpha_{i}\right)}{\sqrt{r-k} \sigma_{k, i}} \\
& =\frac{\sum_{t=R+1}^{R+J}\left(I_{t}^{k, \alpha_{i}}-\alpha_{i}\right)}{\sqrt{(r-k)} \sigma_{k, i}}-\frac{\sum_{t=R+1}^{R+J-(r-k)}\left(I_{t}^{k, \alpha_{i}}-\alpha_{i}\right)}{\sqrt{(r-k)} \sigma_{k, i}} \\
& =\frac{\sqrt{P}}{\sqrt{P m}} \frac{\sum_{t=R+1}^{R+[P s]}\left(I_{t}^{k, \alpha_{i}}-\alpha_{i}\right)}{\sqrt{P} \sigma_{k, i}}-\frac{\sqrt{P}}{\sqrt{P m}} \frac{\sum_{t=R+1}^{R+[P(s-m)]}\left(I_{t}^{k, \alpha_{i}}-\alpha_{i}\right)}{\sqrt{P} \sigma_{k, i}} \\
\underset{P \rightarrow \infty}{\longrightarrow} \frac{1}{\sqrt{m}}(W(s)-W(s-m)) &
\end{aligned}
$$

where $W($.$) is the standard Brownian motion and the limiting distribution, in the last line, is a$ direct consequence of the FCLT. Finally, by the Continuous Mapping Theorem, we have:

$$
\begin{aligned}
& S_{|t|}=\sup _{J}\left|\frac{\sqrt{r-k} \widehat{\alpha}_{i}(J)}{\sigma_{k, \alpha_{i}}}\right| \underset{P \rightarrow \infty}{\longrightarrow} \sup \frac{|W(s)-W(s-m)|}{\sqrt{m}} \\
& A_{|t|}=\frac{1}{P-r+1} \sum_{\underline{J}}\left|\frac{\sqrt{r-k} \widehat{\alpha}_{i}(J)}{\sigma_{k, \alpha_{i}}}\right| \underset{P \rightarrow \infty}{\longrightarrow} \int_{\underline{s}}^{\bar{s}} \frac{|W(s)-W(s-m)|}{\sqrt{m}}
\end{aligned}
$$

where $\underline{J}=[P \underline{s}]$ and $\bar{J}=[P \bar{s}]$. 


\section{Proof of Proposition 2}

Let $\Omega_{k}$ be the variance-covariance matrix of $\mathbf{C}_{\mathbf{k}}(J)$ whose typical element $\omega_{i, j}$ is calculated as follows

$$
\operatorname{cov}\left(c_{k, i}, c_{k, j}\right)=\operatorname{cov}\left(I_{t}^{k, \alpha_{i}}, I_{t}^{k, \alpha_{j}}\right)+\operatorname{cov}\left(I_{t}^{k, \alpha_{i}}, I_{t-k}^{k, \alpha_{j}}\right)+\operatorname{cov}\left(I_{t-k}^{k, \alpha_{i}}, I_{t}^{k, \alpha_{j}}\right)+o(1)
$$

If $i=j$, by Proposition $1, \omega_{i, i}=\operatorname{var}\left(\sqrt{T-k}\left(\widehat{\alpha}_{i}-\alpha_{i}\right)\right)=\alpha_{i}\left(1-\alpha_{i}\right)+2 \alpha_{i}^{3 / 2}\left(1-\alpha_{i}^{1 / 2}\right)$. If $i<j$, $\alpha_{i}<\alpha_{j}$, and we have

$$
\begin{array}{r}
\operatorname{cov}\left(I_{t}^{k, \alpha_{i}}, I_{t}^{k, \alpha_{j}}\right)=E\left(I_{t}^{k, \alpha_{i}} \times I_{t}^{k, \alpha_{j}}\right)-\alpha_{i} \times \alpha_{j}=\alpha_{i}\left(1-\alpha_{j}\right) \\
\operatorname{cov}\left(I_{t}^{k, \alpha_{i}}, I_{t-k}^{k, \alpha_{j}}\right)=E\left(I_{t}^{k, \alpha_{i}} \times I_{t-k}^{k, \alpha_{j}}\right)-\alpha_{i} \times \alpha_{j}=\alpha_{i} \times \alpha_{j}^{1 / 2}-\alpha_{i} \times \alpha_{j} \\
\operatorname{cov}\left(I_{t-k}^{k, \alpha_{i}}, I_{t}^{k, \alpha_{j}}\right)=E\left(I_{t-k}^{k, \alpha_{i}} \times I_{t}^{k, \alpha_{j}}\right)-\alpha_{i} \times \alpha_{j}=\alpha_{i} \times \alpha_{j}^{1 / 2}-\alpha_{i} \times \alpha_{j}
\end{array}
$$

If $i>j$, the above expressions hold by just switching the subindexes $i$ and $j$.

Since the vector $\mathbf{C}_{\mathbf{k}}(J)$ is globally stationary, we can invoke a multivariate FLCT (see Theorem 7.29 and 7.30 in White (2001)). By following the same arguments as in Proposition 1, we have

$$
\begin{aligned}
& \mathbf{W}_{P}(s) \equiv \Omega_{k}^{-1 / 2} \mathbf{C}_{\mathbf{k}}(J) \\
& \underset{P \rightarrow \infty}{\longrightarrow} \frac{1}{\sqrt{m}}(\mathbf{W}(s)-\mathbf{W}(s-m))
\end{aligned}
$$

where $\mathbf{W}(\mathrm{s})$ is a $C$-variate Brownian process. By the Continuous Mapping Theorem, we have

$$
\begin{aligned}
S_{C} & =\sup _{J} \mathbf{C}_{k}(J)^{\prime} \Omega_{k}^{-1} \mathbf{C}_{k}(J) \\
& \underset{P \rightarrow \infty}{\longrightarrow} \sup _{s \in[m, 1]} \frac{(\mathbf{W}(s)-\mathbf{W}(s-m))^{\prime}(\mathbf{W}(s)-\mathbf{W}(s-m))}{m}
\end{aligned}
$$


and

$$
\begin{aligned}
& A_{C}=\frac{1}{P-r+1} \sum_{\underline{J}}^{\bar{J}}\left|\mathbf{C}_{k}(J)^{\prime} \Omega_{k}^{-1} \mathbf{C}_{k}(J)\right| \\
& \underset{P \rightarrow \infty}{\longrightarrow} \int_{\underline{s}}^{\bar{s}} \frac{(\mathbf{W}(r)-\mathbf{W}(r-m))^{\prime}(\mathbf{W}(r)-\mathbf{W}(r-m))}{m}
\end{aligned}
$$

Proof of Proposition 3: Let $\Lambda_{\alpha_{i}}$ be the variance-covariance matrix of $\mathbf{L}_{\alpha_{\mathbf{i}}}(J)$ whose typical element $\lambda_{j, k}$ is calculated as

$$
\lambda_{j, k}=\left\{\begin{array}{cc}
\alpha_{i}\left(1-\alpha_{i}\right)+2 \alpha_{i}^{3 / 2}\left(1-\alpha_{i}^{1 / 2}\right) & \text { if } j=k \\
4 \alpha_{i}^{3 / 2}\left(1-\alpha_{i}^{1 / 2}\right) & \text { if } j \neq k
\end{array}\right.
$$

Therefore, the vector $\mathbf{L}_{\alpha_{\mathbf{i}}}(J)$ is globally stationary, and we can invoke a multivariate FLCT (see Theorems 7.29 and 7.30 in White (2001)). By following the same arguments as in Proposition 1, we have

$$
\begin{aligned}
& \mathbf{W}_{P}(s) \equiv \Lambda_{\alpha_{i}}^{-1 / 2} \mathbf{L}_{\alpha_{\mathbf{i}}}(J) \\
& \underset{P \rightarrow \infty}{\longrightarrow} \frac{1}{\sqrt{m}}(\mathbf{W}(s)-\mathbf{W}(s-m))
\end{aligned}
$$

where $\mathbf{W}(s)$ is a $L$-variate Brownian process. By the Continuous Mapping Theorem, we have

$$
\begin{aligned}
S_{L} & =\sup _{J} \mathbf{L}_{\alpha_{\mathbf{i}}}(J)^{\prime} \Lambda_{\alpha_{i}}^{-1} \mathbf{L}_{\alpha_{\mathbf{i}}}(J) \\
& \longrightarrow \sup _{P \rightarrow \infty} \frac{(\mathbf{W}(s)-\mathbf{W}(s-m))^{\prime}(\mathbf{W}(s)-\mathbf{W}(s-m))}{m}
\end{aligned}
$$




$$
\begin{aligned}
& A_{L}=\frac{1}{P-r+1} \sum_{\underline{J}}^{\bar{J}} \mathbf{L}_{\alpha_{\mathbf{i}}}(J)^{\prime} \Lambda_{\alpha_{i}}^{-1} \mathbf{L}_{\alpha_{\mathbf{i}}}(J) \\
& \underset{P \rightarrow \infty}{\longrightarrow} \int_{\underline{s}}^{\bar{s}} \frac{(\mathbf{W}(s)-\mathbf{W}(s-m))^{\prime}(\mathbf{W}(s)-\mathbf{W}(s-m))}{m}
\end{aligned}
$$




\section{References}

[1] Andrews, D.W. (1993), "Tests for Parameter Instability and Structural Change with Unknown Change Point", Econometrica, Vol. 61, No. 4, pp. 821-856.

[2] Andrews, D.W. and W. Ploberger (1994), "Optimal Tests when a Nuisance Parameter is Present only under the Alternative", Econometrica Vol. 62, No. 6, pp. 1383-1414.

[3] Chow, G.C. (1960), "Tests of Equality between Sets of Coefficients in Two Linear Regressions", Econometrica, Vol. 28, pp. 591-605.

[4] Diebold, F., A. Gunther, and A. Tay (1998), "Evaluating Density Forecasts with Applications to Financial Risk Management", International Economic Review, Vol. 39, No. 4, pp. 863-883.

[5] Elliott, G. and U. Muller (2007), "Confidence Sets for the Date of a Single Break in Linear Time Series Regressions", Journal of Econometrics, Vol.141, pp. 1196-1218.

[6] Amisano, G. and R. Giacomini (2007), "Comparing Density Forecast via Weighted Likelihood Ratio Tests", Journal of Business and Economic Statistics, Vol. 25, No. 2, pp. 177-190.

[7] Giacomini, R. and B. Rossi (2009), "Detecting and Predicting Forecast Breakdowns", Review of Economic Studies, Vol. 76, No. 2, pp. 669-705.

[8] Giacomini, R. and B. Rossi (2010), "Forecast Comparisons in Unstable Environments", Journal of Applied Econometrics, Vol. 25, No. 4, pp. 595-620.

[9] González-Rivera, G., Z. Senyuz, and E. Yoldas (2011), "Autocontours: Dynamic Specification Testing", Journal of Business and Economic Statistics, Vol. 29, No. 1, pp. 186-200.

[10] González-Rivera, G. and E. Yoldas (2012), "Autocontour-based Evaluation of Multivariate Predictive Densities", International Journal of Forecasting, Vol. 28, No. 2, pp. 328-342.

[11] González-Rivera, G. and Y. Sun (2014), "Generalized Autocontours: Evaluation of Multivariate Density Models", International Journal of Forecasting, Forthcoming. 
[12] Goyal, A. and I. Welch (2003), "Predicting the Equity Premium with Dividend Ratios", Management Science, Vol. 49, pp. 639-654.

[13] Paye, B. and A. Timmermann (2006), "Instability of Return Prediction Models", Journal of Empirical Finance, Vol. 13, No. 3, pp. 274-315.

[14] Pesaran, M.H. and A. Timmermann (2002), "Market Timing and Return Prediction Under Model Instability", Journal of Empirical Finance, Vol. 9, No. 5, pp. 495-510.

[15] Pesaran, M. H., D. Pettenuzzo, and A. Timmermann (2006), "Forecasting Time Series Subject to Multiple Structural Breaks", Review of Economic Studies, Vol. 73, pp. 1057-1084.

[16] Pesaran, M.H. and A. Timmermann (2007), "Selection of Estimation Window in the Presence of Breaks", Journal of Econometrics, Vol. 137, No. 1, pp. 134-161.

[17] Rapach, D.E. and D. Zhou (2014), "Forecasting Stock Returns", Handbook of Economic Forecasting, Vol. 2, pp. 328-383.

[18] Rogoff, K.S. and V. Stavrakeva, V. (2008), "The Continuing Puzzle of Short Horizon Exchange Rate Forecasting", NBER Working Papers 14071

[19] Rossi, B. (2006), "Are Exchange Rates Really Random Walks? Some Evidence Robust to Parameter Instability", Macroeconomic Dynamics, Vol. 10, No. 1, pp. 20-38.

[20] Rossi, B. (2014), "Advances in Forecasting under Instability", Handbook of Economic Forecasting, Vol. 2, pp. 1203-1324.

[21] Rossi, B. and T. Sekhposyan,T. (2010), "Have Economic Models Forecasting Performance for US Output Growth and Inflation Changed Over Time, and When?", International Journal of Forecasting, Vol. 26, No.4, pp. 808835.

[22] Rossi, B. and T. Sekhposyan,T. (2011), "Forecast Optimality Tests in the Presence of Instabilities", ERID Working paper No. 109.

[23] Stock, J. H., and M.W. Watson (1999), "Forecasting Inflation", Journal of Monetary Economics, Vol. 44, pp. 293-335. 
[24] Stock, J.H. and M.W. Watson (2003), "Forecasting Output and Inflation: The Role of Asset Prices", Journal of Economic Literature, pp. 788-829.

[25] White, H. (2000), "Asymptotic Theory for Econometricians, Revised Edition", Academic Press. 


\section{Tables: Asymptotic Distributions}

In the following six tables, the percentiles are obtained from 2000 replications with a sample size of 20,000 observations in each replication.

\begin{tabular}{|c|c|c|c|c|c|c|c|c|c|}
\hline \multicolumn{10}{|c|}{ Asymptotic Distribution of $S_{|t|}$ Statistic } \\
\hline \multicolumn{10}{|c|}{$m$} \\
\hline Percentile & 0.1 & 0.2 & 0.3 & 0.4 & 0.5 & 0.6 & 0.7 & 0.8 & 0.9 \\
\hline $99 \%$ & 3.950 & 3.713 & 3.510 & 3.396 & 3.428 & 3.213 & 3.089 & 3.112 & 2.951 \\
\hline $95 \%$ & 3.502 & 3.267 & 3.066 & 2.926 & 2.866 & 2.679 & 2.537 & 2.439 & 2.406 \\
\hline $90 \%$ & 3.292 & 2.987 & 2.845 & 2.642 & 2.571 & 2.361 & 2.240 & 2.121 & 2.015 \\
\hline $80 \%$ & 3.020 & 2.684 & 2.522 & 2.372 & 2.217 & 2.053 & 1.935 & 1.849 & 1.655 \\
\hline $70 \%$ & 2.843 & 2.489 & 2.315 & 2.156 & 1.999 & 1.834 & 1.697 & 1.582 & 1.392 \\
\hline $60 \%$ & 2.702 & 2.343 & 2.145 & 1.983 & 1.819 & 1.653 & 1.529 & 1.383 & 1.197 \\
\hline $50 \%$ & 2.569 & 2.203 & 2.015 & 1.830 & 1.664 & 1.515 & 1.365 & 1.221 & 1.021 \\
\hline $40 \%$ & 2.457 & 2.090 & 1.884 & 1.684 & 1.515 & 1.373 & 1.212 & 1.066 & 0.867 \\
\hline $30 \%$ & 2.339 & 1.975 & 1.747 & 1.540 & 1.379 & 1.228 & 1.071 & 0.915 & 0.730 \\
\hline $20 \%$ & 2.201 & 1.822 & 1.586 & 1.384 & 1.226 & 1.074 & 0.923 & 0.791 & 0.606 \\
\hline $10 \%$ & 2.033 & 1.629 & 1.420 & 1.199 & 1.055 & 0.901 & 0.761 & 0.631 & 0.479 \\
\hline $5 \%$ & 1.922 & 1.492 & 1.290 & 1.065 & 0.943 & 0.813 & 0.663 & 0.535 & 0.394 \\
\hline $1 \%$ & 1.730 & 1.265 & 1.072 & 0.829 & 0.739 & 0.631 & 0.498 & 0.409 & 0.287 \\
\hline \multicolumn{10}{|c|}{ Asymptotic Distribution of $A_{|t|}$ Statistic } \\
\hline \multicolumn{10}{|c|}{$m$} \\
\hline Percentile & 0.1 & 0.2 & 0.3 & 0.4 & 0.5 & 0.6 & 0.7 & 0.8 & 0.9 \\
\hline $99 \%$ & 1.241 & 1.435 & 1.656 & 1.824 & 2.204 & 2.181 & 2.360 & 2.542 & 2.583 \\
\hline $95 \%$ & 1.078 & 1.199 & 1.355 & 1.487 & 1.697 & 1.694 & 1.774 & 1.854 & 1.998 \\
\hline $90 \%$ & 1.004 & 1.088 & 1.206 & 1.300 & 1.418 & 1.406 & 1.490 & 1.586 & 1.666 \\
\hline $80 \%$ & 0.918 & 0.970 & 1.035 & 1.101 & 1.115 & 1.128 & 1.183 & 1.264 & 1.256 \\
\hline $70 \%$ & 0.870 & 0.891 & 0.922 & 0.948 & 0.939 & 0.928 & 0.966 & 1.017 & 1.019 \\
\hline $60 \%$ & 0.825 & 0.825 & 0.827 & 0.837 & 0.818 & 0.796 & 0.807 & 0.824 & 0.825 \\
\hline $50 \%$ & 0.789 & 0.760 & 0.760 & 0.741 & 0.710 & 0.685 & 0.682 & 0.675 & 0.661 \\
\hline $40 \%$ & 0.752 & 0.706 & 0.690 & 0.653 & 0.615 & 0.581 & 0.551 & 0.534 & 0.507 \\
\hline $30 \%$ & 0.711 & 0.649 & 0.617 & 0.568 & 0.524 & 0.487 & 0.453 & 0.417 & 0.378 \\
\hline $20 \%$ & 0.673 & 0.589 & 0.544 & 0.497 & 0.451 & 0.409 & 0.358 & 0.320 & 0.274 \\
\hline $10 \%$ & 0.608 & 0.517 & 0.465 & 0.409 & 0.359 & 0.319 & 0.269 & 0.234 & 0.186 \\
\hline $5 \%$ & 0.567 & 0.458 & 0.413 & 0.342 & 0.310 & 0.260 & 0.219 & 0.188 & 0.140 \\
\hline $1 \%$ & 0.478 & 0.381 & 0.325 & 0.251 & 0.235 & 0.200 & 0.152 & 0.135 & 0.096 \\
\hline
\end{tabular}

Table 1: Asymptotic Distributions of $S_{|t|}$ and $A_{|t|}$ Statistics 


\begin{tabular}{|c|c|c|c|c|c|c|c|c|c|}
\hline \multicolumn{10}{|c|}{ Asymptotic Distribution of $S_{C}$ Statistic } \\
\hline \multicolumn{10}{|c|}{$m$} \\
\hline Percentile & 0.1 & 0.2 & 0.3 & 0.4 & 0.5 & 0.6 & 0.7 & 0.8 & 0.9 \\
\hline $99 \%$ & 42.488 & 39.592 & 38.534 & 37.694 & 37.361 & 35.925 & 36.051 & 34.591 & 31.804 \\
\hline $95 \%$ & 37.159 & 34.956 & 32.983 & 32.277 & 30.902 & 29.597 & 29.008 & 27.773 & 25.962 \\
\hline $90 \%$ & 35.155 & 32.562 & 30.722 & 29.378 & 28.069 & 27.176 & 26.181 & 24.685 & 23.356 \\
\hline $80 \%$ & 32.624 & 29.964 & 28.071 & 26.673 & 25.329 & 24.182 & 22.935 & 21.621 & 20.175 \\
\hline $70 \%$ & 30.932 & 28.330 & 26.359 & 24.906 & 23.421 & 22.132 & 20.932 & 19.423 & 18.125 \\
\hline $60 \%$ & 29.689 & 26.991 & 24.908 & 23.416 & 21.858 & 20.579 & 19.179 & 17.901 & 16.550 \\
\hline $50 \%$ & 28.540 & 25.798 & 23.648 & 22.111 & 20.626 & 19.136 & 17.708 & 16.501 & 15.241 \\
\hline $40 \%$ & 27.482 & 24.522 & 22.468 & 20.900 & 19.254 & 17.672 & 16.440 & 15.111 & 13.829 \\
\hline $30 \%$ & 26.508 & 23.385 & 21.197 & 19.510 & 17.873 & 16.413 & 15.058 & 13.685 & 12.477 \\
\hline $20 \%$ & 25.351 & 22.174 & 19.836 & 18.193 & 16.514 & 14.927 & 13.582 & 12.250 & 10.898 \\
\hline $10 \%$ & 23.731 & 20.400 & 18.067 & 16.333 & 14.568 & 13.061 & 11.741 & 10.480 & 8.987 \\
\hline $5 \%$ & 22.376 & 18.902 & 16.821 & 14.879 & 13.338 & 11.876 & 10.383 & 9.037 & 7.852 \\
\hline $1 \%$ & 20.394 & 16.833 & 14.737 & 12.807 & 11.208 & 9.884 & 8.275 & 6.929 & 5.905 \\
\hline \multicolumn{10}{|c|}{ Asymptotic Distribution of $A_{C}$ Statistic } \\
\hline \multicolumn{10}{|c|}{$m$} \\
\hline Percentile & 0.1 & 0.2 & 0.3 & 0.4 & 0.5 & 0.6 & 0.7 & 0.8 & 0.9 \\
\hline $99 \%$ & 16.342 & 18.610 & 20.265 & 22.301 & 24.658 & 26.534 & 27.422 & 28.202 & 27.987 \\
\hline $95 \%$ & 15.298 & 16.576 & 17.794 & 18.927 & 19.924 & 20.838 & 21.866 & 22.084 & 22.533 \\
\hline $90 \%$ & 14.762 & 15.734 & 16.456 & 17.287 & 18.022 & 18.629 & 18.996 & 19.612 & 19.965 \\
\hline $80 \%$ & 14.099 & 14.648 & 15.143 & 15.477 & 16.014 & 16.449 & 16.761 & 16.975 & 17.096 \\
\hline $70 \%$ & 13.672 & 14.029 & 14.209 & 14.439 & 14.705 & 14.937 & 15.107 & 15.111 & 15.290 \\
\hline $60 \%$ & 13.295 & 13.393 & 13.478 & 13.566 & 13.597 & 13.552 & 13.629 & 13.790 & 13.772 \\
\hline $50 \%$ & 12.955 & 12.873 & 12.847 & 12.820 & 12.666 & 12.591 & 12.534 & 12.514 & 12.547 \\
\hline $40 \%$ & 12.623 & 12.388 & 12.178 & 12.014 & 11.722 & 11.471 & 11.290 & 11.362 & 11.311 \\
\hline $30 \%$ & 12.262 & 11.851 & 11.535 & 11.227 & 10.909 & 10.576 & 10.364 & 10.166 & 10.073 \\
\hline $20 \%$ & 11.828 & 11.264 & 10.837 & 10.423 & 9.991 & 9.555 & 9.288 & 8.965 & 8.786 \\
\hline $10 \%$ & 11.298 & 10.483 & 9.871 & 9.330 & 8.746 & 8.297 & 7.860 & 7.558 & 7.143 \\
\hline $5 \%$ & 10.840 & 9.895 & 9.120 & 8.436 & 7.849 & 7.358 & 6.750 & 6.380 & 5.967 \\
\hline $1 \%$ & 10.030 & 8.791 & 7.993 & 7.279 & 6.363 & 5.659 & 5.069 & 4.629 & 4.382 \\
\hline
\end{tabular}

Table 2: Asymptotic Distribution of $S_{C}$ and $A_{C}$ Statistics (13 autocontours) 


\begin{tabular}{|c|c|c|c|c|c|c|c|c|c|}
\hline \multicolumn{10}{|c|}{ Asymptotic Distribution of $S_{L}$ Statistic } \\
\hline \multicolumn{10}{|c|}{$m$} \\
\hline percentile & 0.1 & 0.2 & 0.3 & 0.4 & 0.5 & 0.6 & 0.7 & 0.8 & 0.9 \\
\hline $99 \%$ & 26.825 & 25.069 & 23.492 & 23.010 & 22.057 & 20.621 & 19.801 & 19.370 & 17.756 \\
\hline $95 \%$ & 22.719 & 21.121 & 19.941 & 18.372 & 17.199 & 16.042 & 15.645 & 14.470 & 13.631 \\
\hline $90 \%$ & 20.867 & 19.109 & 17.489 & 16.318 & 15.203 & 14.241 & 13.301 & 12.552 & 11.636 \\
\hline $80 \%$ & 18.859 & 16.979 & 15.273 & 14.045 & 12.859 & 11.973 & 11.213 & 10.267 & 9.325 \\
\hline $70 \%$ & 17.452 & 15.505 & 13.917 & 12.671 & 11.665 & 10.585 & 9.708 & 8.788 & 7.949 \\
\hline $60 \%$ & 16.389 & 14.279 & 12.920 & 11.587 & 10.492 & 9.537 & 8.610 & 7.765 & 6.927 \\
\hline $50 \%$ & 14.810 & 12.425 & 11.052 & 9.796 & 8.827 & 7.916 & 6.830 & 6.071 & 5.192 \\
\hline $40 \%$ & 15.534 & 13.300 & 11.910 & 10.616 & 9.576 & 8.678 & 7.698 & 6.917 & 6.068 \\
\hline $30 \%$ & 13.970 & 11.675 & 10.164 & 8.891 & 7.999 & 6.948 & 6.048 & 5.213 & 4.423 \\
\hline $20 \%$ & 13.066 & 10.697 & 9.249 & 8.023 & 7.039 & 6.054 & 5.113 & 4.388 & 3.646 \\
\hline $10 \%$ & 11.896 & 9.535 & 8.158 & 7.011 & 5.863 & 4.966 & 4.213 & 3.484 & 2.743 \\
\hline $5 \%$ & 11.145 & 8.544 & 7.070 & 6.093 & 5.099 & 4.286 & 3.492 & 2.786 & 2.187 \\
\hline $1 \%$ & 9.583 & 7.267 & 5.857 & 4.821 & 3.950 & 3.173 & 2.585 & 1.907 & 1.510 \\
\hline \multicolumn{10}{|c|}{ Asymptotic Distribution of $A_{L}$ Statistic } \\
\hline \multicolumn{10}{|c|}{$m$} \\
\hline percentile & 0.1 & 0.2 & 0.3 & 0.4 & 0.5 & 0.6 & 0.7 & 0.8 & 0.9 \\
\hline $99 \%$ & 7.260 & 8.419 & 9.620 & 10.837 & 12.021 & 12.943 & 13.509 & 14.458 & 14.629 \\
\hline $95 \%$ & 6.507 & 7.336 & 7.948 & 8.396 & 9.045 & 9.636 & 10.116 & 10.569 & 10.913 \\
\hline $90 \%$ & 6.122 & 6.673 & 7.034 & 7.503 & 7.961 & 8.313 & 8.562 & 8.929 & 9.220 \\
\hline $80 \%$ & 5.705 & 6.038 & 6.259 & 6.452 & 6.643 & 6.822 & 6.995 & 7.104 & 7.309 \\
\hline $70 \%$ & 5.383 & 5.583 & 5.669 & 5.733 & 5.820 & 5.868 & 5.929 & 5.977 & 6.040 \\
\hline $60 \%$ & 5.164 & 5.208 & 5.200 & 5.198 & 5.212 & 5.158 & 5.191 & 5.122 & 5.128 \\
\hline $50 \%$ & 4.742 & 4.604 & 4.468 & 4.361 & 4.224 & 4.021 & 3.885 & 3.817 & 3.767 \\
\hline $40 \%$ & 4.951 & 4.890 & 4.831 & 4.770 & 4.663 & 4.570 & 4.506 & 4.452 & 4.438 \\
\hline $30 \%$ & 4.535 & 4.316 & 4.123 & 3.922 & 3.717 & 3.476 & 3.324 & 3.174 & 3.088 \\
\hline $20 \%$ & 4.294 & 3.988 & 3.712 & 3.418 & 3.174 & 2.973 & 2.715 & 2.584 & 2.480 \\
\hline $10 \%$ & 4.017 & 3.539 & 3.189 & 2.922 & 2.602 & 2.320 & 2.086 & 1.927 & 1.746 \\
\hline $5 \%$ & 3.752 & 3.237 & 2.867 & 2.508 & 2.246 & 1.921 & 1.695 & 1.459 & 1.292 \\
\hline $1 \%$ & 3.337 & 2.681 & 2.222 & 1.875 & 1.621 & 1.388 & 1.150 & 0.960 & 0.777 \\
\hline
\end{tabular}

Table 3: Asymptotic Distribution of $S_{L}$ and $A_{L}$ Statistics (five lags) 


\begin{tabular}{|c|c|c|c|c|c|c|c|c|c|c|c|c|c|}
\hline Fixed Scheme & $S_{|t|}^{l, 1}$ & $S_{|t|}^{l, 2}$ & $S_{|t|}^{l, 3}$ & $S_{|t|}^{l, 4}$ & $S_{|t|}^{l, 5}$ & $S_{|t|}^{l, 6}$ & $S_{|t|}^{l, 7}$ & $S_{|t|}^{l, 8}$ & $S_{|t|}^{l, 9}$ & $S_{|t|}^{l, 10}$ & $S_{|t|}^{l, 11}$ & $S_{|t|}^{l, 12}$ & $S_{|t|}^{l, 13}$ \\
\hline$l=1$ & 0.14 & 0.45 & 0.765 & 0.956 & 0.979 & 0.987 & 0.995 & 0.992 & 0.987 & 0.976 & 0.938 & 0.867 & 0.589 \\
\hline$l=2$ & 0.14 & 0.464 & 0.789 & 0.955 & 0.981 & 0.99 & 0.993 & 0.993 & 0.988 & 0.974 & 0.943 & 0.864 & 0.593 \\
\hline$l=3$ & 0.14 & 0.419 & 0.792 & 0.965 & 0.985 & 0.997 & 0.997 & 0.994 & 0.985 & 0.971 & 0.937 & 0.871 & 0.594 \\
\hline$l=4$ & 0.11 & 0.439 & 0.797 & 0.953 & 0.986 & 0.993 & 0.994 & 0.994 & 0.99 & 0.979 & 0.938 & 0.866 & 0.595 \\
\hline \multirow{2}{*}{$l=5$} & 0.12 & 0.453 & 0.796 & 0.952 & 0.984 & 0.992 & 0.994 & 0.993 & 0.989 & 0.971 & 0.938 & 0.867 & 0.598 \\
\hline & $A_{|t|}^{l, 1}$ & $A_{|t|}^{l, 2}$ & $A_{|t|}^{l, 3}$ & $A_{|t|}^{l, 4}$ & $A_{|t|}^{l, 5}$ & $A_{|t|}^{l, 6}$ & $A_{|t|}^{1,7}$ & $A_{|t|}^{l, 8}$ & $A_{|t|}^{l, 9}$ & $A_{|t|}^{l, 10}$ & $A_{|t|}^{l, 11}$ & $A_{|t|}^{l, 12}$ & $A_{|t|}^{l, 13}$ \\
\hline$l=1$ & 0.08 & 0.244 & 0.698 & 0.898 & 0.948 & 0.969 & 0.981 & 0.978 & 0.968 & 0.945 & 0.904 & 0.812 & 0.588 \\
\hline$l=2$ & 0.09 & 0.277 & 0.741 & 0.896 & 0.952 & 0.965 & 0.979 & 0.983 & 0.972 & 0.947 & 0.898 & 0.815 & 0.585 \\
\hline$l=3$ & 0.07 & 0.276 & 0.72 & 0.893 & 0.941 & 0.969 & 0.98 & 0.984 & 0.967 & 0.943 & 0.906 & 0.815 & 0.588 \\
\hline$l=4$ & 0.07 & 0.319 & 0.702 & 0.887 & 0.957 & 0.971 & 0.979 & 0.981 & 0.971 & 0.944 & 0.904 & 0.817 & 0.586 \\
\hline \multirow[t]{2}{*}{$l=5$} & 0.08 & 0.339 & 0.722 & 0.906 & 0.951 & 0.968 & 0.985 & 0.976 & 0.972 & 0.944 & 0.903 & 0.816 & 0.584 \\
\hline & $S_{C}^{l, 13}$ & $A_{C}^{l, 13}$ & & & & & & & & & & & \\
\hline$l=1$ & 0.961 & 0.921 & & & & & & & & & & & \\
\hline$l=2$ & 0.965 & 0.924 & & & & & & & & & & & \\
\hline$l=3$ & 0.967 & 0.923 & & & & & & & & & & & \\
\hline$l=4$ & 0.963 & 0.921 & & & & & & & & & & & \\
\hline$l=5$ & 0.965 & 0.921 & & & & & & & & & & & \\
\hline \multirow[b]{2}{*}{$C=7$} & $S_{L}^{2,7}$ & $S_{L}^{3,7}$ & $S_{L}^{4,7}$ & $S_{L}^{5,7}$ & $A_{L}^{2,7}$ & $A_{L}^{3,7}$ & $A_{L}^{4,7}$ & $A_{L}^{5,7}$ & & & & & \\
\hline & 0.989 & 0.985 & 0.976 & 0.964 & 0.98 & 0.969 & 0.956 & 0.942 & & & & & \\
\hline Rolling Scheme & $S_{|t|}^{l, 1}$ & $S_{|t|}^{l, 2}$ & $S_{|t|}^{l, 3}$ & $S_{|t|}^{l, 4}$ & $S_{|t|}^{l, 5}$ & $S_{|t|}^{l, 6}$ & $S_{|t|}^{l, 7}$ & $S_{|t|}^{l, 8}$ & $S_{|t|}^{l, 9}$ & $S_{|t|}^{l, 10}$ & $S_{|t|}^{l, 11}$ & $S_{|t|}^{l, 12}$ & $S_{|t|}^{l, 13}$ \\
\hline$l=1$ & 0.18 & 0.12 & 0.308 & 0.416 & 0.459 & 0.394 & 0.42 & 0.264 & 0.21 & 0.08 & 0.119 & 0.092 & 0.08 \\
\hline$l=2$ & 0.11 & 0.11 & 0.295 & 0.324 & 0.323 & 0.431 & 0.433 & 0.333 & 0.193 & 0.084 & 0.123 & 0.093 & 0.08 \\
\hline$l=3$ & 0.11 & 0.11 & 0.258 & 0.402 & 0.386 & 0.39 & 0.472 & 0.325 & 0.207 & 0.096 & 0.123 & 0.094 & 0.09 \\
\hline$l=4$ & 0.13 & 0.11 & 0.289 & 0.439 & 0.41 & 0.413 & 0.378 & 0.325 & 0.173 & 0.093 & 0.121 & 0.092 & 0.082 \\
\hline \multirow[t]{2}{*}{$l=5$} & 0.16 & 0.11 & 0.262 & 0.445 & 0.418 & 0.423 & 0.438 & 0.318 & 0.171 & 0.091 & 0.121 & 0.092 & 0.083 \\
\hline & $A_{|t|}^{l, 1}$ & $A_{|t|}^{l, 2}$ & $A_{|t|}^{l, 3}$ & $A_{|t|}^{l, 4}$ & $A_{|t|}^{l, 5}$ & $A_{|t|}^{l, 6}$ & $A_{|t|}^{l, 7}$ & $A_{|t|}^{l, 8}$ & $A_{|t|}^{l, 9}$ & $A_{|t|}^{l, 10}$ & $A_{|t|}^{l, 11}$ & $A_{|t|}^{l, 12}$ & $A_{|t|}^{l, 13}$ \\
\hline$l=1$ & 0.25 & 0.318 & 0.559 & 0.783 & 0.738 & 0.710 & 0.534 & 0.634 & 0.418 & 0.18 & 0.21 & 0.102 & 0.091 \\
\hline$l=2$ & 0.22 & 0.292 & 0.527 & 0.733 & 0.71 & 0.714 & 0.56 & 0.621 & 0.438 & 0.17 & 0.216 & 0.100 & 0.092 \\
\hline$l=3$ & 0.24 & 0.275 & 0.521 & 0.768 & 0.756 & 0.744 & 0.499 & 0.638 & 0.442 & 0.155 & 0.224 & 0.103 & 0.092 \\
\hline$l=4$ & 0.25 & 0.28 & 0.519 & 0.806 & 0.775 & 0.759 & 0.611 & 0.638 & 0.421 & 0.133 & 0.21 & 0.101 & 0.092 \\
\hline \multirow[t]{2}{*}{$l=5$} & 0.25 & 0.305 & 0.516 & 0.797 & 0.781 & 0.769 & 0.533 & 0.626 & 0.392 & 0.121 & 0.24 & 0.101 & 0.093 \\
\hline & $S_{C}^{l, 13,}$ & $A_{C}^{l, 13}$ & & & & & & & & & & & \\
\hline$l=1$ & 0.638 & 0.749 & & & & & & & & & & & \\
\hline$l=2$ & 0.521 & 0.733 & & & & & & & & & & & \\
\hline$l=3$ & 0.619 & 0.801 & & & & & & & & & & & \\
\hline$l=4$ & 0.641 & 0.822 & & & & & & & & & & & \\
\hline$l=5$ & 0.637 & 0.803 & & & & & & & & & & & \\
\hline$C=7$ & $\begin{array}{c}S_{L}^{2,7} \\
0.414\end{array}$ & $\begin{array}{c}S_{L}^{3,7} \\
0.419\end{array}$ & $\begin{array}{c}S_{L}^{4,7} \\
0.408\end{array}$ & $\begin{array}{c}S_{L}^{5,7} \\
0.432\end{array}$ & $\begin{array}{c}A_{L}^{2,7} \\
0.563\end{array}$ & $\begin{array}{l}A_{L}^{3,7} \\
0.59\end{array}$ & $\begin{array}{c}A_{L}^{4,7} \\
0.578\end{array}$ & $\begin{array}{c}A_{L}^{5,7} \\
0.532\end{array}$ & & & & & \\
\hline \multicolumn{14}{|c|}{$\begin{array}{l}S_{L}^{l, 7}, A_{L}^{l, 7} \text { stacking lags up to } l=2, \ldots .5 \text { and considering the } 50 \% \text { autocontour. } \\
S_{C}^{l, 13} \text { and } A_{C}^{l, 13} \text { stacking all } 13 \text { autocontours for one lag } l=1,2,3,4,5 . \\
1000 \text { Monte Carlo replications and } 500 \text { bootstrap samples. } \\
\mathrm{T}=650, \mathrm{R}=350 . \mathrm{P}=300, m=1 / 3, \text { and break point at } R+\tau P \text { for } \tau=1 / 3\end{array}$} \\
\hline
\end{tabular}

Table 6: Power for DGP1 under Fixed and Rolling Schemes 


\begin{tabular}{|c|c|c|c|c|c|c|c|c|c|c|c|c|c|}
\hline Fixed Scheme & $S_{|t|}^{l, 1}$ & $S_{|t|}^{l, 2}$ & $S_{|t|}^{l, 3}$ & $S_{|t|}^{l, 4}$ & $S_{|t|}^{l, 5}$ & $S_{|t|}^{l, 6}$ & $S_{|t|}^{l, 7}$ & $S_{|t|}^{l, 8}$ & $S_{|t|}^{l, 9}$ & $S_{|t|}^{l, 10}$ & $S_{|t|}^{l, 11}$ & $S_{|t|}^{l, 12}$ & $S_{|t|}^{l, 13}$ \\
\hline$l=1$ & 0.292 & 0.271 & 0.191 & 0.084 & 0.073 & 0.109 & 0.281 & 0.278 & 0.391 & 0.512 & 0.638 & 0.638 & 0.518 \\
\hline$l=2$ & 0.26 & 0.239 & 0.171 & 0.079 & 0.075 & 0.106 & 0.282 & 0.292 & 0.418 & 0.518 & 0.637 & 0.639 & 0.52 \\
\hline$l=3$ & 0.285 & 0.265 & 0.175 & 0.089 & 0.075 & 0.12 & 0.283 & 0.29 & 0.404 & 0.53 & 0.635 & 0.639 & 0.523 \\
\hline$l=4$ & 0.311 & 0.26 & 0.162 & 0.088 & 0.063 & 0.096 & 0.282 & 0.284 & 0.399 & 0.512 & 0.624 & 0.636 & 0.526 \\
\hline \multirow[t]{2}{*}{$l=5$} & 0.273 & 0.253 & 0.158 & 0.068 & 0.068 & 0.108 & 0.282 & 0.288 & 0.408 & 0.539 & 0.645 & 0.638 & 0.531 \\
\hline & $A_{|t|}^{l, 1}$ & $A_{|t|}^{l, 2}$ & $A_{|t|}^{l, 3}$ & $A_{|t|}^{l, 4}$ & $A_{|t|}^{l, 5}$ & $A_{|t|}^{\ell, 6}$ & $A_{|t|}^{l, 7}$ & $A_{|t|}^{l, 8}$ & $A_{|t|}^{l, 9}$ & $A_{|t|}^{l, 10}$ & $A_{|t|}^{l, 11}$ & $A_{|t|}^{l, 12}$ & $A_{|t|}^{l, 13}$ \\
\hline$l=1$ & 0.345 & 0.242 & 0.162 & 0.09 & 0.078 & 0.127 & 0.275 & 0.271 & 0.361 & 0.472 & 0.561 & 0.604 & 0.527 \\
\hline$l=2$ & 0.327 & 0.225 & 0.16 & 0.081 & 0.077 & 0.12 & 0.269 & 0.277 & 0.377 & 0.467 & 0.56 & 0.606 & 0.522 \\
\hline$l=3$ & 0.347 & 0.239 & 0.167 & 0.089 & 0.087 & 0.123 & 0.272 & 0.286 & 0.374 & 0.468 & 0.558 & 0.6 & 0.527 \\
\hline$l=4$ & 0.368 & 0.246 & 0.159 & 0.09 & 0.069 & 0.111 & 0.272 & 0.277 & 0.345 & 0.455 & 0.55 & 0.592 & 0.523 \\
\hline \multirow[t]{2}{*}{$l=5$} & 0.317 & 0.222 & 0.146 & 0.086 & 0.072 & 0.12 & 0.273 & 0.288 & 0.37 & 0.469 & 0.564 & 0.6 & 0.523 \\
\hline & $S_{C}^{l, 13}$ & $A_{C}^{l, 13}$ & & & & & & & & & & & \\
\hline$l=1$ & 0.659 & 0.595 & & & & & & & & & & & \\
\hline$l=2$ & 0.651 & 0.604 & & & & & & & & & & & \\
\hline$l=3$ & 0.666 & 0.625 & & & & & & & & & & & \\
\hline$l=4$ & 0.644 & 0.625 & & & & & & & & & & & \\
\hline$l=5$ & 0.652 & 0.611 & & & & & & & & & & & \\
\hline$C=7$ & $\begin{array}{c}S_{L}^{2,7} \\
0.286\end{array}$ & $\begin{array}{c}S_{L}^{3,7} \\
0.285\end{array}$ & $\begin{array}{c}S_{L}^{4,7} \\
0.281\end{array}$ & $\begin{array}{c}S_{L}^{5,7} \\
0.285\end{array}$ & $\begin{array}{c}A_{L}^{2,7} \\
0.271\end{array}$ & $\begin{array}{c}A_{L}^{3,7} \\
0.273\end{array}$ & $\begin{array}{c}A_{L}^{4,7} \\
0.276\end{array}$ & $\begin{array}{c}A_{L}^{5,7} \\
0.275\end{array}$ & & & & & \\
\hline Rolling Scheme & $S_{|t|}^{l, 1}$ & $S_{|t|}^{l, 2}$ & $S_{|t|}^{l, 3}$ & $S_{|t|}^{l, 4}$ & $S_{|t|}^{l, 5}$ & $S_{|t|}^{l, 6}$ & $S_{|t|}^{l, 7}$ & $S_{|t|}^{l, 8}$ & $S_{|t|}^{l, 9}$ & $S_{|t|}^{l, 10}$ & $S_{|t|}^{l, 11}$ & $S_{|t|}^{l, 12}$ & $S_{|t|}^{l, 13}$ \\
\hline$l=1$ & 0.116 & 0.104 & 0.088 & 0.063 & 0.05 & 0.056 & 0.112 & 0.11 & 0.171 & 0.341 & 0.302 & 0.264 & 0.261 \\
\hline$l=2$ & 0.114 & 0.09 & 0.086 & 0.063 & 0.054 & 0.051 & 0.118 & 0.113 & 0.172 & 0.318 & 0.296 & 0.264 & 0.262 \\
\hline$l=3$ & 0.107 & 0.094 & 0.076 & 0.065 & 0.06 & 0.053 & 0.108 & 0.126 & 0.189 & 0.335 & 0.28 & 0.273 & 0.266 \\
\hline$l=4$ & 0.113 & 0.104 & 0.072 & 0.054 & 0.058 & 0.049 & 0.175 & 0.114 & 0.167 & 0.329 & 0.287 & 0.266 & 0.267 \\
\hline \multirow{2}{*}{$l=5$} & 0.109 & 0.093 & 0.08 & 0.053 & 0.05 & 0.053 & 0.16 & 0.121 & 0.184 & 0.337 & 0.274 & 0.265 & 0.267 \\
\hline & $A_{|t|}^{l, 1}$ & $A_{|t|}^{l, 2}$ & $A_{|t|}^{l, 3}$ & $A_{|t|}^{l, 4}$ & $A_{|t|}^{l, 5}$ & $A_{|t|}^{l, 6}$ & $A_{|t|}^{l, 7}$ & $A_{|t|}^{l, 8}$ & $A_{|t|}^{l, 9}$ & $A_{|t|}^{l, 10}$ & $A_{|t|}^{l, 11}$ & $A_{|t|}^{l, 12}$ & $A_{|t|}^{l, 13}$ \\
\hline$l=1$ & 0.179 & 0.129 & 0.099 & 0.078 & 0.066 & 0.075 & 0.19 & 0.151 & 0.204 & 0.36 & 0.344 & 0.35 & 0.378 \\
\hline$l=2$ & 0.169 & 0.112 & 0.082 & 0.075 & 0.066 & 0.076 & 0.18 & 0.145 & 0.205 & 0.386 & 0.345 & 0.343 & 0.379 \\
\hline$l=3$ & 0.175 & 0.139 & 0.092 & 0.07 & 0.067 & 0.077 & 0.196 & 0.159 & 0.228 & 0.378 & 0.352 & 0.348 & 0.378 \\
\hline$l=4$ & 0.183 & 0.143 & 0.083 & 0.063 & 0.066 & 0.077 & 0.192 & 0.144 & 0.201 & 0.358 & 0.342 & 0.352 & 0.375 \\
\hline \multirow[t]{2}{*}{$l=5$} & 0.159 & 0.129 & 0.09 & 0.063 & 0.066 & 0.065 & 0.191 & 0.151 & 0.208 & 0.371 & 0.347 & 0.35 & 0.377 \\
\hline & $S_{C}^{l, 13}$ & $A_{C}^{l, 13}$ & & & & & & & & & & & \\
\hline$l=1$ & 0.409 & 0.414 & & & & & & & & & & & \\
\hline$l=2$ & 0.363 & 0.394 & & & & & & & & & & & \\
\hline$l=3$ & 0.381 & 0.427 & & & & & & & & & & & \\
\hline$l=4$ & 0.398 & 0.431 & & & & & & & & & & & \\
\hline$l=5$ & 0.306 & 0.318 & & & & & & & & & & & \\
\hline$C=7$ & $\begin{array}{c}S_{L}^{2,7} \\
0.168\end{array}$ & $\begin{array}{c}S_{L}^{3,7} \\
0.115\end{array}$ & $\begin{array}{c}S_{L}^{4,7} \\
0.113\end{array}$ & $\begin{array}{c}S_{L}^{5,7} \\
0.112\end{array}$ & $\begin{array}{c}A_{L}^{2,7} \\
0.129\end{array}$ & $\begin{array}{c}A_{L}^{3,7} \\
0.189\end{array}$ & $\begin{array}{c}A_{L}^{4,7} \\
0.191\end{array}$ & $\begin{array}{c}A_{L}^{5,7} \\
0.182\end{array}$ & & & & & \\
\hline
\end{tabular}

Notes: 13 autocontours $C=[0.01,0.05,0.1,0.2,0.3,0.4,0.5,0.6,0.7,0.8,0.9,0.95,0.99]$.

$S_{|t|}^{l, 7}, A_{|t|}^{l, 7}$ for $l=1,2, \ldots 5 ; 7$ refers to the $50 \%$ autocontour.

$S_{L}^{l, 7}, A_{L}^{l, 7}$ stacking lags up to $l=2, \ldots .5$ and considering the $50 \%$ autocontour.

$S_{C}^{l, 13}$ and $A_{C}^{l, 13}$ stacking all 13 autocontours for one lag $l=1,2,3,4,5$.

1000 Monte Carlo replications and 500 bootstrap samples.

$\mathrm{T}=650, \mathrm{R}=350, \mathrm{P}=300, m=1 / 3$, and break point at $R+\tau P$ for $\tau=1 / 3$

Table 7: Power for DGP2 under Fixed and Rolling Schemes 


\begin{tabular}{|c|c|c|c|c|c|c|c|c|c|c|c|c|c|}
\hline Fixed Scheme & $S_{|t|}^{l, 1}$ & $S_{|t|}^{l, 2}$ & $S_{|t|}^{l, 3}$ & $S_{|t|}^{l, 4}$ & $S_{|t|}^{l, 5}$ & $S_{|t|}^{l, 6}$ & $S_{|t|}^{l, 7}$ & $S_{|t|}^{l, 8}$ & $S_{|t|}^{l, 9}$ & $S_{|t|}^{l, 10}$ & $S_{|t|}^{l, 11}$ & $S_{|t|}^{l, 12}$ & $S_{|t|}^{l, 13}$ \\
\hline$l=1$ & 0.95 & 0.998 & 0.999 & 0.999 & 0.999 & 1 & 0.97 & 0.985 & 0.961 & 0.849 & 0.449 & 0.33 & 0.29 \\
\hline$l=2$ & 0.96 & 0.998 & 0.999 & 1 & 0.999 & 0.997 & 0.982 & 0.981 & 0.96 & 0.859 & 0.444 & 0.16 & 0.19 \\
\hline$l=3$ & 0.97 & 0.997 & 0.998 & 1 & 0.999 & 0.998 & 0.979 & 0.982 & 0.956 & 0.85 & 0.436 & 0.35 & 0.31 \\
\hline$l=4$ & 0.96 & 0.997 & 0.998 & 1 & 0.998 & 0.998 & 0.98 & 0.984 & 0.965 & 0.853 & 0.446 & 0.36 & 0.13 \\
\hline \multirow[t]{2}{*}{$l=5$} & 0.96 & 0.997 & 1 & 0.999 & 1 & 0.999 & 0.982 & 0.983 & 0.96 & 0.854 & 0.447 & 0.2 & 0.23 \\
\hline & $A_{|t|}^{l, 1}$ & $A_{|t|}^{l, 2}$ & $A_{|t|}^{l, 3}$ & $A_{|t|}^{l, 4}$ & $A_{|t|}^{l, 5}$ & $A_{|t|}^{l, 6}$ & $A_{|t|}^{1,7}$ & $A_{|t|}^{l, 8}$ & $A_{|t|}^{l, 9}$ & $A_{|t|}^{l, 10}$ & $A_{|t|}^{l, 11}$ & $A_{|t|}^{l, 12}$ & $A_{|t|}^{l, 13}$ \\
\hline$l=1$ & 0.93 & 0.951 & 1 & 0.999 & 0.999 & 0.995 & 0.95 & 0.973 & 0.914 & 0.475 & 0.38 & 0.25 & 0.16 \\
\hline$l=2$ & 0.931 & 0.967 & 0.998 & 1 & 0.999 & 0.994 & 0.952 & 0.974 & 0.896 & 0.518 & 0.34 & 0.22 & 0.17 \\
\hline$l=3$ & 0.937 & 0.98 & 0.998 & 0.999 & 0.997 & 0.995 & 0.95 & 0.973 & 0.883 & 0.555 & 0.36 & 0.24 & 0.19 \\
\hline$l=4$ & 0.935 & 0.96 & 1 & 0.998 & 0.997 & 0.994 & 0.95 & 0.968 & 0.908 & 0.487 & 0.31 & 0.32 & 0.22 \\
\hline \multirow[t]{2}{*}{$l=5$} & 0.939 & 0.95 & 0.999 & 0.998 & 0.997 & 0.995 & 0.95 & 0.962 & 0.875 & 0.545 & 0.31 & 0.12 & 0.23 \\
\hline & $S_{C}^{l, 13}$ & $A_{C}^{l, 13}$ & & & & & & & & & & & \\
\hline$l=1$ & 1 & 0.97 & & & & & & & & & & & \\
\hline$l=2$ & 1 & 0.98 & & & & & & & & & & & \\
\hline$l=3$ & 0.999 & 0.97 & & & & & & & & & & & \\
\hline$l=4$ & 1 & 0.999 & & & & & & & & & & & \\
\hline$l=5$ & 1 & 1 & & & & & & & & & & & \\
\hline \multirow[b]{2}{*}{$C=7$} & $S_{L}^{2,7}$ & $S_{L}^{3,7}$ & $S_{L}^{4,7}$ & $S_{L}^{5,7}$ & $A_{L}^{2,7}$ & $A_{L}^{3,7}$ & $A_{L}^{4,7}$ & $A_{L}^{5,7}$ & & & & & \\
\hline & 0.98 & 0.98 & 0.98 & 0.98 & 0.97 & 0.969 & 0.97 & 0.97 & & & & & \\
\hline Rolling Scheme & $S_{|t|}^{l, 1}$ & $S_{|t|}^{l, 2}$ & $S_{|t|}^{l, 3}$ & $S_{|t|}^{l, 4}$ & $S_{|t|}^{l, 5}$ & $S_{|t|}^{l, 6}$ & $S_{|t|}^{l, 7}$ & $S_{|t|}^{l, 8}$ & $S_{|t|}^{l, 9}$ & $S_{|t|}^{l, 10}$ & $S_{|t|}^{l, 11}$ & $S_{|t|}^{l, 12}$ & $S_{|t|}^{l, 13}$ \\
\hline$l=1$ & 0.46 & 0.461 & 0.395 & 0.184 & 0.26 & 0.329 & 0.396 & 0.457 & 0.401 & 0.134 & 0.212 & 0.092 & 0.08 \\
\hline$l=2$ & 0.358 & 0.32 & 0.338 & 0.381 & 0.421 & 0.475 & 0.511 & 0.508 & 0.39 & 0.121 & 0.241 & 0.093 & 0.08 \\
\hline$l=3$ & 0.437 & 0.398 & 0.389 & 0.442 & 0.465 & 0.524 & 0.524 & 0.518 & 0.385 & 0.099 & 0.223 & 0.094 & 0.09 \\
\hline$l=4$ & 0.445 & 0.425 & 0.444 & 0.481 & 0.502 & 0.544 & 0.54 & 0.512 & 0.345 & 0.095 & 0.232 & 0.092 & 0.082 \\
\hline \multirow[t]{2}{*}{$l=5$} & 0.448 & 0.409 & 0.425 & 0.468 & 0.511 & 0.54 & 0.546 & 0.506 & 0.361 & 0.075 & 0.232 & 0.092 & 0.083 \\
\hline & $A_{|t|}^{l, 1}$ & $A_{|t|}^{l, 2}$ & $A_{|t|}^{l, 3}$ & $A_{|t|}^{l, 4}$ & $A_{|t|}^{l, 5}$ & $A_{|t|}^{l, 6}$ & $A_{|t|}^{l, 7}$ & $A_{|t|}^{l, 8}$ & $A_{|t|}^{l, 9}$ & $A_{|t|}^{l, 10}$ & $A_{|t|}^{l, 11}$ & $A_{|t|}^{l, 12}$ & $A_{|t|}^{l, 13}$ \\
\hline$l=1$ & 0.189 & 0.219 & 0.332 & 0.473 & 0.584 & 0.678 & 0.726 & 0.739 & 0.706 & 0.395 & 0.163 & 0.082 & 0.06 \\
\hline$l=2$ & 0.585 & 0.628 & 0.686 & 0.788 & 0.808 & 0.834 & 0.826 & 0.815 & 0.716 & 0.356 & 0.171 & 0.081 & 0.06 \\
\hline$l=3$ & 0.68 & 0.711 & 0.728 & 0.796 & 0.832 & 0.839 & 0.832 & 0.811 & 0.7 & 0.334 & 0.163 & 0.081 & 0.07 \\
\hline$l=4$ & 0.684 & 0.733 & 0.765 & 0.832 & 0.851 & 0.861 & 0.846 & 0.815 & 0.698 & 0.308 & 0.162 & 0.081 & 0.062 \\
\hline \multirow[t]{2}{*}{$l=5$} & 0.674 & 0.717 & 0.762 & 0.82 & 0.846 & 0.86 & 0.848 & 0.814 & 0.669 & 0.291 & 0.161 & 0.081 & 0.063 \\
\hline & $S_{C}^{l, 13}$ & $A_{C}^{l, 13}$ & & & & & & & & & & & \\
\hline$l=1$ & 0.57 & 0.817 & & & & & & & & & & & \\
\hline$l=2$ & 0.539 & 0.845 & & & & & & & & & & & \\
\hline$l=3$ & 0.53 & 0.814 & & & & & & & & & & & \\
\hline$l=4$ & 0.532 & 0.78 & & & & & & & & & & & \\
\hline \multirow[t]{3}{*}{$l=5$} & 0.534 & 0.782 & & & & & & & & & & & \\
\hline & $S_{L}^{2,7}$ & $S_{L}^{3,7}$ & $S_{L}^{4,7}$ & $S_{L}^{5,7}$ & $A_{L}^{2,7}$ & $A_{L}^{3,7}$ & $A_{L}^{4,7}$ & $A_{L}^{5,7}$ & & & & & \\
\hline & 0.477 & 0.496 & 0.48 & 0.421 & 0.728 & 0.75 & 0.749 & 0.703 & & & & & \\
\hline
\end{tabular}

Notes: 13 autocontours $C=[0.01,0.05,0.1,0.2,0.3,0.4,0.5,0.6,0.7,0.8,0.9,0.95,0.99]$.

$S_{|t|}^{l, 7}, A_{|t|}^{l, 7}$ for $l=1,2, \ldots 5 ; 7$ refers to the $50 \%$ autocontour.

$S_{L}^{l, 7}, A_{L}^{l, 7}$ stacking lags up to $l=2, \ldots .5$ and considering the $50 \%$ autocontour.

$S_{C}^{l, 13}$ and $A_{C}^{l, 13}$ stacking all 13 autocontours for one lag $l=1,2,3,4,5$.

1000 Monte Carlo replications and 500 bootstrap samples.

$\mathrm{T}=650, \mathrm{R}=350, \mathrm{P}=300, m=1 / 3$, and break point at $R+\tau P$ for $\tau=1 / 3$

Table 8: Power for DGP3 under Fixed and Rolling Schemes 


\begin{tabular}{|c|c|c|c|c|c|c|c|c|c|c|c|c|c|}
\hline Fixed Scheme & $S_{|t|}^{l, 1}$ & $S_{|t|}^{l, 2}$ & $S_{|t|}^{l, 3}$ & $S_{|t|}^{l, 4}$ & $S_{|t|}^{l, 5}$ & $S_{|t|}^{l, 6}$ & $S_{|t|}^{l, 7}$ & $S_{|t|}^{l, 8}$ & $S_{|t|}^{l, 9}$ & $S_{|t|}^{l, 10}$ & $S_{|t|}^{l, 11}$ & $S_{|t|}^{l, 12}$ & $S_{|t|}^{l, 13}$ \\
\hline$l=1$ & 1 & 1 & 0.999 & 1 & 1 & 1 & 0.999 & 0.999 & 0.989 & 0.984 & 0.969 & 0.93 & 0.79 \\
\hline$l=2$ & 0.999 & 1 & 0.999 & 0.996 & 1 & 1 & 1 & 1 & 0.98 & 0.983 & 0.967 & 0.901 & 0.78 \\
\hline$l=3$ & 0.998 & 1 & 1 & 1 & 1 & 0.999 & 1 & 1 & 0.996 & 0.98 & 0.965 & 0.912 & 0.781 \\
\hline$l=4$ & 1 & 0.999 & 1 & 1 & 0.998 & 1 & 0.999 & 1 & 0.99 & 0.98 & 0.962 & 0.923 & 0.744 \\
\hline \multirow[t]{2}{*}{$l=5$} & 1 & 0.996 & 1 & 1 & 1 & 1 & 1 & 0.998 & 1 & 0.98 & 0.966 & 0.917 & 0.775 \\
\hline & $A_{|t|}^{l, 1}$ & $A_{|t|}^{l, 2}$ & $A_{|t|}^{l, 3}$ & $A_{|t|}^{l, 4}$ & $A_{|t|}^{l, 5}$ & $A_{|t|}^{l, 6}$ & $A_{|t|}^{l, 7}$ & $A_{|t|}^{l, 8}$ & $A_{|t|}^{l, 9}$ & $A_{|t|}^{l, 10}$ & $A_{|t|}^{l, 11}$ & $A_{|t|}^{l, 12}$ & $A_{|t|}^{l, 13}$ \\
\hline$l=1$ & 0.989 & 1 & 0.995 & 1 & 1 & 1 & 0.98 & 0.998 & 0.989 & 0.96 & 0.914 & 0.812 & 0.639 \\
\hline$l=2$ & 0.989 & 1 & 0.995 & 0.995 & 0.997 & 1 & 0.988 & 0.989 & 0.986 & 0.967 & 0.934 & 0.815 & 0.633 \\
\hline$l=3$ & 0.99 & 1 & 1 & 1 & 1 & 0.997 & 0.985 & 0.99 & 0.986 & 0.966 & 0.914 & 0.815 & 0.622 \\
\hline$l=4$ & 1 & 0.996 & 1 & 1 & 0.996 & $0 .$. & 0.989 & 0.991 & 0.986 & 0.967 & 0.954 & 0.817 & 0.644 \\
\hline$l=5$ & 1 & 0.996 & 1 & 1 & 1 & 1 & 0.989 & 0.996 & 0.986 & 0.97 & 0.914 & 0.816 & 0.675 \\
\hline $\begin{aligned} l & =1 \\
l & =2 \\
l & =3 \\
l & =4 \\
l & =5\end{aligned}$ & $\begin{array}{c}S_{C}^{l, 13} \\
1 \\
1 \\
1 \\
1 \\
1\end{array}$ & $\begin{array}{c}A_{C}^{l, 13} \\
1 \\
1 \\
1 \\
1 \\
1 \\
\end{array}$ & & & & & & & & & & & \\
\hline$C=7$ & $\begin{array}{c}S_{L}^{2,7} \\
1 \\
\end{array}$ & $\begin{array}{c}S_{L}^{3,7} \\
1 \\
\end{array}$ & $\begin{array}{c}S_{L}^{4,7} \\
1 \\
\end{array}$ & $\begin{array}{c}S_{L}^{5,7} \\
1 \\
\end{array}$ & $\begin{array}{c}A_{L}^{2,7} \\
1 \\
\end{array}$ & $\begin{array}{c}A_{L}^{3,7} \\
1\end{array}$ & $\begin{array}{c}A_{L}^{4,7} \\
1 \\
\end{array}$ & $\begin{array}{c}A_{L}^{5,7} \\
1 \\
\end{array}$ & & & & & \\
\hline Rolling Scheme & $S_{|t|}^{l, 1}$ & $S_{|t|}^{l, 2}$ & $S_{|t|}^{l, 3}$ & $S_{|t|}^{l, 4}$ & $S_{|t|}^{l, 5}$ & $S_{|t|}^{l, 6}$ & $S_{|t|}^{l, 7}$ & $S_{|t|}^{l, 8}$ & $S_{|t|}^{l, 9}$ & $S_{|t|}^{l, 10}$ & $S_{|t|}^{l, 11}$ & $S_{|t|}^{l, 12}$ & $S_{|t|}^{l, 13}$ \\
\hline$l=1$ & 0.569 & 0.461 & 0.486 & 0.589 & 0.696 & 0.746 & 0.76 & 0.729 & 0.69 & 0.643 & 0.481 & 0.331 & 0.123 \\
\hline$l=2$ & 0.44 & 0.387 & 0.358 & 0.577 & 0.664 & 0.73 & 0.744 & 0.733 & 0.703 & 0.629 & 0.464 & 0.332 & 0.12 \\
\hline$l=3$ & 0.541 & 0.465 & 0.421 & 0.528 & 0.649 & 0.695 & 0.705 & 0.71 & 0.691 & 0.624 & 0.445 & 0.332 & 0.127 \\
\hline$l=4$ & 0.586 & 0.514 & 0.48 & 0.525 & 0.639 & 0.698 & 0.703 & 0.694 & 0.663 & 0.618 & 0.447 & 0.319 & 0.131 \\
\hline \multirow[t]{2}{*}{$l=5$} & 0.571 & 0.495 & 0.484 & 0.525 & 0.643 & 0.724 & 0.722 & 0.723 & 0.678 & 0.627 & 0.448 & 0.318 & 0.135 \\
\hline & $A_{|t|}^{l, 1}$ & $A_{|t|}^{l, 2}$ & $A_{|t|}^{l, 3}$ & $A_{|t|}^{l, 4}$ & $A_{|t|}^{l, 5}$ & $A_{|t|}^{l, 6}$ & $A_{|t|}^{l, 7}$ & $A_{|t|}^{l, 8}$ & $A_{|t|}^{l, 9}$ & $A_{|t|}^{l, 10}$ & $A_{|t|}^{l, 11}$ & $A_{|t|}^{l, 12}$ & $A_{|t|}^{l, 13}$ \\
\hline$l=1$ & 0.25 & 0.318 & 0.559 & 0.761 & 0.831 & 0.861 & 0.858 & 0.839 & 0.819 & 0.763 & 0.606 & 0.461 & 0.236 \\
\hline$l=2$ & 0.22 & 0.292 & 0.527 & 0.739 & 0.794 & 0.835 & 0.857 & 0.852 & 0.82 & 0.767 & 0.601 & 0.46 & 0.242 \\
\hline$l=3$ & 0.24 & 0.275 & 0.521 & 0.705 & 0.789 & 0.835 & 0.85 & 0.854 & 0.819 & 0.767 & 0.609 & 0.45 & 0.237 \\
\hline$l=4$ & 0.25 & 0.28 & 0.519 & 0.699 & 0.795 & 0.833 & 0.86 & 0.842 & 0.8 & 0.736 & 0.606 & 0.443 & 0.24 \\
\hline \multirow[t]{2}{*}{$l=5$} & 0.25 & 0.305 & 0.516 & 0.704 & 0.789 & 0.837 & 0.846 & 0.848 & 0.823 & 0.75 & 0.588 & 0.446 & 0.242 \\
\hline & $S_{C}^{l, 13}$ & $A_{C}^{l, 13}$ & & & & & & & & & & & \\
\hline$l=1$ & 0.75 & 0.85 & & & & & & & & & & & \\
\hline$l=2$ & 0.74 & 0.85 & & & & & & & & & & & \\
\hline$l=3$ & 0.76 & 0.87 & & & & & & & & & & & \\
\hline$l=4$ & 0.76 & 0.83 & & & & & & & & & & & \\
\hline$l=5$ & 0.771 & 0.81 & & & & & & & & & & & \\
\hline$C=7$ & $\begin{array}{c}S_{L}^{2,7} \\
0.656\end{array}$ & $\begin{array}{c}S_{L}^{3,7} \\
0.558\end{array}$ & $\begin{array}{c}S_{L}^{4,7} \\
0.558\end{array}$ & $\begin{array}{l}S_{L}^{5,7} \\
0.56\end{array}$ & $\begin{array}{c}A_{L}^{2,7} \\
0.801\end{array}$ & $\begin{array}{c}A_{L}^{3,7} \\
0.724\end{array}$ & $\begin{array}{c}A_{L}^{4,7} \\
0.656\end{array}$ & $\begin{array}{c}A_{L}^{5,7} \\
0.605\end{array}$ & & & & & \\
\hline
\end{tabular}

Notes: 13 autocontours $C=[0.01,0.05,0.1,0.2,0.3,0.4,0.5,0.6,0.7,0.8,0.9,0.95,0.99]$.

$S_{|t|}^{l, 7}, A_{|t|}^{l, 7}$ for $l=1,2, \ldots 5 ; 7$ refers to the $50 \%$ autocontour.

$S_{L}^{l, 7}, A_{L}^{l, 7}$ stacking lags up to $l=2, \ldots .5$ and considering the $50 \%$ autocontour.

$S_{C}^{l, 13}$ and $A_{C}^{l, 13}$ stacking all 13 autocontours for one lag $l=1,2,3,4,5$.

1000 Monte Carlo replications and 500 bootstrap samples.

$\mathrm{T}=650, \mathrm{R}=350, \mathrm{P}=300, m=1 / 3$, and break point at $R+\tau P$ for $\tau=1 / 3$

Table 9: Power for DGP4 under Fixed and Rolling Schemes 


\section{Tables: Phillips Curve}

\begin{tabular}{|c|c|c|c|c|c|c|c|c|c|c|c|c|c|}
\hline Fixed Scheme & $S_{|t|}^{l, 1}$ & $S_{|t|}^{l, 2}$ & $S_{|t|}^{l, 3}$ & $S_{|t|}^{l, 4}$ & $S_{|t|}^{l, 5}$ & $S_{|t|}^{l, 6}$ & $S_{|t|}^{l, 7}$ & $S_{|t|}^{l, 8}$ & $S_{|t|}^{l, 9}$ & $S_{|t|}^{l, 10}$ & $S_{|t|}^{l, 11}$ & $S_{|t|}^{l, 12}$ & $S_{|t|}^{l, 13}$ \\
\hline$l=1$ & 0.017 & 0.529 & 0.523 & 0.471 & 0.268 & 0.002 & 0.008 & 0.045 & 0.136 & 0.091 & 0.085 & 0.031 & 0.0001 \\
\hline$l=2$ & 0.015 & 0.285 & 0.55 & 0.434 & 0.481 & 0.002 & 0.005 & 0.041 & 0.157 & 0.086 & 0.09 & 0.027 & 0.0001 \\
\hline$l=3$ & 0.028 & 0.584 & 0.614 & 0.651 & 0.419 & 0.006 & 0.005 & 0.03 & 0.049 & 0.088 & 0.073 & 0.013 & 0.0001 \\
\hline$l=4$ & 0.013 & 0.104 & 0.807 & 0.285 & 0.209 & 0.009 & 0.003 & 0.027 & 0.098 & 0.088 & 0.069 & 0.012 & 0.0001 \\
\hline \multirow[t]{2}{*}{$l=5$} & 0.031 & 0.221 & 0.212 & 0.302 & 0.206 & 0.009 & 0.006 & 0.034 & 0.103 & 0.088 & 0.057 & 0.006 & 0.0001 \\
\hline & $A_{|t|}^{l, 1}$ & $A_{|t|}^{l, 2}$ & $A_{|t|}^{l, 3}$ & $A_{|t|}^{l, 4}$ & $A_{|t|}^{l, 5}$ & $A_{|t|}^{l, 6}$ & $A_{|t|}^{l, 7}$ & $A_{|t|}^{l, 8}$ & $A_{|t|}^{l, 9}$ & $A_{|t|}^{l, 10}$ & $A_{|t|}^{l, 11}$ & $A_{|t|}^{l, 12}$ & $A_{|t|}^{l, 13}$ \\
\hline$l=1$ & 0.342 & 0.671 & 0.759 & 0.405 & 0.163 & 0.042 & 0.013 & 0.248 & 0.243 & 0.243 & 0.412 & 0.199 & 0.042 \\
\hline$l=2$ & 0.335 & 0.453 & 0.621 & 0.352 & 0.972 & 0.043 & 0.022 & 0.336 & 0.297 & 0.223 & 0.332 & 0.25 & 0.04 \\
\hline$l=3$ & 0.494 & 0.579 & 0.316 & 0.567 & 0.309 & 0.041 & 0.016 & 0.216 & 0.225 & 0.235 & 0.359 & 0.197 & 0.049 \\
\hline$l=4$ & 0.338 & 0.348 & 0.764 & 0.319 & 0.152 & 0.033 & 0.024 & 0.144 & 0.264 & 0.223 & 0.327 & 0.226 & 0.042 \\
\hline \multirow[t]{2}{*}{$l=5$} & 0.138 & 0.595 & 0.637 & 0.238 & 0.142 & 0.032 & 0.024 & 0.218 & 0.273 & 0.222 & 0.308 & 0.184 & 0.042 \\
\hline & $S_{C}^{l, 13}$ & $A_{C}^{l, 13}$ & & & & & & & & & & & \\
\hline$l=1$ & 0.004 & 0.022 & & & & & & & & & & & \\
\hline$l=2$ & 0.001 & 0.019 & & & & & & & & & & & \\
\hline$l=3$ & 0.003 & 0.016 & & & & & & & & & & & \\
\hline$l=4$ & 0.001 & 0.025 & & & & & & & & & & & \\
\hline$l=5$ & 0.001 & 0.025 & & & & & & & & & & & \\
\hline & $S_{L}^{2,7}$ & $S_{L}^{3,7}$ & $S_{L}^{4,7}$ & $S_{L}^{5,7}$ & $A_{L}^{2,7}$ & $A_{L}^{3,7}$ & $A_{L}^{4,7}$ & $A_{L}^{5,7}$ & & & & & \\
\hline$C=7$ & 0.006 & 0.009 & 0.012 & 0.01 & 0.027 & 0.018 & 0.027 & 0.029 & & & & & \\
\hline
\end{tabular}

\begin{tabular}{|c|c|c|c|c|c|c|c|c|c|c|c|c|c|}
\hline Rolling Scheme & $S_{|t|}^{l, 1}$ & $S_{|t|}^{l, 2}$ & $S_{|t|}^{l, 3}$ & $S_{|t|}^{l, 4}$ & $S_{|t|}^{l, 5}$ & $S_{|t|}^{l, 6}$ & $S_{|t|}^{l, 7}$ & $S_{|t|}^{l, 8}$ & $S_{|t|}^{l, 9}$ & $S_{|t|}^{l, 10}$ & $S_{|t|}^{l, 11}$ & $S_{|t|}^{l, 12}$ & $S_{|t|}^{l, 13}$ \\
\hline$l=1$ & 0.528 & 0.46 & 0.843 & 0.472 & 0.142 & 0.002 & 0.001 & 0.007 & 0.008 & 0.093 & 0.281 & 0.026 & 0.0002 \\
\hline$l=2$ & 0.258 & 0.35 & 0.58 & 0.251 & 0.229 & 0.007 & 0.004 & 0.009 & 0.002 & 0.081 & 0.24 & 0.024 & 0.0003 \\
\hline$l=3$ & 0.559 & 0.31 & 0.741 & 0.437 & 0.16 & 0.002 & 0.001 & 0.002 & 0.002 & 0.066 & 0.288 & 0.013 & 0.0003 \\
\hline$l=4$ & 0.38 & 0.31 & 0.479 & 0.463 & 0.17 & 0.001 & 0.001 & 0.003 & 0.008 & 0.088 & 0.275 & 0.021 & 0.0002 \\
\hline \multirow[t]{2}{*}{$l=5$} & 0.285 & 0.249 & 0.297 & 0.225 & 0.12 & 0.007 & 0.001 & 0.003 & 0.003 & 0.063 & 0.327 & 0.019 & 0.0002 \\
\hline & $A_{|t|}^{l, 1}$ & $A_{|t|}^{l, 2}$ & $A_{|t|}^{l, 3}$ & $A_{|t|}^{l, 4}$ & $A_{|t|}^{l, 5}$ & $A_{|t|}^{l, 6}$ & $A_{|t|}^{l, 7}$ & $A_{|t|}^{l, 8}$ & $A_{|t|}^{l, 9}$ & $A_{|t|}^{l, 10}$ & $A_{|t|}^{l, 11}$ & $A_{|t|}^{l, 12}$ & $A_{|t|}^{l, 13}$ \\
\hline$l=1$ & 0.789 & 0.801 & 0.853 & 0.656 & 0.143 & 0.001 & 0.023 & 0.033 & 0.032 & 0.145 & 0.297 & 0.271 & 0.024 \\
\hline$l=2$ & 0.267 & 0.55 & 0.812 & 0.244 & 0.568 & 0.002 & 0.018 & 0.024 & 0.045 & 0.127 & 0.283 & 0.337 & 0.037 \\
\hline$l=3$ & 0.751 & 0.918 & 0.923 & 0.723 & 0.144 & 0.007 & 0.015 & 0.032 & 0.047 & 0.114 & 0.288 & 0.24 & 0.023 \\
\hline$l=4$ & 0.358 & 0.476 & 0.575 & 0.448 & 0.108 & 0.001 & 0.015 & 0.031 & 0.031 & 0.142 & 0.313 & 0.271 & 0.034 \\
\hline \multirow[t]{2}{*}{$l=5$} & 0.305 & 0.655 & 0.576 & 0.251 & 0.129 & 0.009 & 0.018 & 0.031 & 0.039 & 0.13 & 0.286 & 0.288 & 0.033 \\
\hline & $S_{C}^{l, 13}$ & $A_{C}^{l, 13}$ & & & & & & & & & & & \\
\hline$l=1$ & 0.001 & 0.009 & & & & & & & & & & & \\
\hline$l=2$ & 0.0003 & 0.004 & & & & & & & & & & & \\
\hline$l=3$ & 0.0003 & 0.01 & & & & & & & & & & & \\
\hline$l=4$ & 0.0003 & 0.005 & & & & & & & & & & & \\
\hline$l=5$ & 0.002 & 0.004 & & & & & & & & & & & \\
\hline & $S_{L}^{2,7}$ & $S_{L}^{3,7}$ & $S_{L}^{4,7}$ & $S_{L}^{5,7}$ & $A_{L}^{2,7}$ & $A_{L}^{3,7}$ & $A_{L}^{4,7}$ & $A_{L}^{5,7}$ & & & & & \\
\hline$C=7$ & 0.008 & 0.008 & 0.006 & 0.009 & 0.038 & 0.025 & 0.019 & 0.031 & & & & & \\
\hline
\end{tabular}

Notes: 13 autocontours $C=[0.01,0.05,0.1,0.2,0.3,0.4,0.5,0.6,0.7,0.8,0.9,0.95,0.99]$.

$S_{|t|}^{l, 7}, A_{|t|}^{l, 7}$ for $l=1,2, \ldots 5 ; 7$ refers to the $50 \%$ autocontour.

$S_{L}^{l, 7}, A_{L}^{l, 7}$ stacking lags up to $l=2, \ldots .5$ and considering the $50 \%$ autocontour.

$S_{C}^{l, 13}$ and $A_{C}^{l, 13}$ stacking all 13 autocontours for one lag $l=1,2,3,4,5$.

500 bootstrap samples. $\mathrm{T}=649, \mathrm{R}=360, \mathrm{P}=289, m=0.69$

Table 10: Bootstrapped P-values: Linear Phillips Curve (Fixed and Rolling Schemes) 


\begin{tabular}{|c|c|c|c|c|c|c|c|c|c|c|c|c|c|}
\hline Model 2 & $S_{|t|}^{l, 1}$ & $S_{|t|}^{l, 2}$ & $S_{|t|}^{l, 3}$ & $S_{|t|}^{l, 4}$ & $S_{|t|}^{l, 5}$ & $S_{|t|}^{l, 6}$ & $S_{|t|}^{l, 7}$ & $S_{|t|}^{l, 8}$ & $S_{|t|}^{l, 9}$ & $S_{|t|}^{l, 10}$ & $S_{|t|}^{l, 11}$ & $S_{|t|}^{l, 12}$ & $S_{|t|}^{l, 13}$ \\
\hline$l=1$ & 0.542 & 0.682 & 0.306 & 0.254 & 0.18 & 0.196 & 0.178 & 0.26 & 0.4 & 0.188 & 0.044 & 0.026 & 0.036 \\
\hline$l=2$ & 0.478 & 0.32 & 0.226 & 0.15 & 0.102 & 0.118 & 0.102 & 0.116 & 0.228 & 0.088 & 0.034 & 0.024 & 0.032 \\
\hline$l=3$ & 0.802 & 0.394 & 0.188 & 0.236 & 0.172 & 0.176 & 0.146 & 0.234 & 0.274 & 0.192 & 0.038 & 0.024 & 0.032 \\
\hline$l=4$ & 0.496 & 0.65 & 0.524 & 0.296 & 0.188 & 0.2 & 0.19 & 0.284 & 0.422 & 0.21 & 0.042 & 0.024 & 0.034 \\
\hline \multirow[t]{2}{*}{$l=5$} & 0.794 & 0.19 & 0.286 & 0.186 & 0.136 & 0.148 & 0.164 & 0.296 & 0.386 & 0.166 & 0.032 & 0.024 & 0.032 \\
\hline & $A_{|t|}^{l, 1}$ & $A_{|t|}^{l, 2}$ & $A_{|t|}^{l, 3}$ & $A_{|t|}^{l, 4}$ & $A_{|t|}^{l, 5}$ & $A_{|t|}^{l, 6}$ & $A_{|t|}^{l, 7}$ & $A_{|t|}^{l, 8}$ & $A_{|t|}^{l, 9}$ & $A_{|t|}^{l, 10}$ & $A_{|t|}^{l, 11}$ & $A_{|t|}^{l, 12}$ & $A_{|t|}^{l, 13}$ \\
\hline$l=1$ & 0.496 & 0.628 & 0.296 & 0.264 & 0.176 & 0.198 & 0.178 & 0.288 & 0.424 & 0.256 & 0.046 & 0.028 & 0.036 \\
\hline$l=2$ & 0.438 & 0.298 & 0.216 & 0.152 & 0.102 & 0.116 & 0.104 & 0.16 & 0.27 & 0.128 & 0.044 & 0.026 & 0.032 \\
\hline$l=3$ & 0.372 & 0.374 & 0.298 & 0.24 & 0.168 & 0.17 & 0.148 & 0.246 & 0.288 & 0.22 & 0.046 & 0.026 & 0.032 \\
\hline$l=4$ & 0.442 & 0.416 & 0.366 & 0.33 & 0.21 & 0.2 & 0.21 & 0.31 & 0.434 & 0.24 & 0.046 & 0.026 & 0.034 \\
\hline \multirow[t]{2}{*}{$l=5$} & 0.362 & 0.22 & 0.332 & 0.192 & 0.138 & 0.15 & 0.17 & 0.32 & 0.402 & 0.232 & 0.036 & 0.024 & 0.032 \\
\hline & $S_{C}^{l, 13}$ & $A_{C}^{l, 13}$ & & & & & & & & & & & \\
\hline$l=1$ & 0.328 & 0.332 & & & & & & & & & & & \\
\hline$l=2$ & 0.18 & 0.18 & & & & & & & & & & & \\
\hline$l=3$ & 0.284 & 0.272 & & & & & & & & & & & \\
\hline$l=4$ & 0.32 & 0.328 & & & & & & & & & & & \\
\hline$l=5$ & 0.266 & 0.268 & & & & & & & & & & & \\
\hline$C=7$ & $\begin{array}{c}S_{L}^{2,7} \\
0.112\end{array}$ & $\begin{array}{l}S_{L}^{3,7} \\
0.12\end{array}$ & $\begin{array}{c}S_{L}^{4,7} \\
0.12\end{array}$ & $\begin{array}{c}S_{L}^{5,7} \\
0.116\end{array}$ & $\begin{array}{c}A_{L}^{2,7} \\
0.118\end{array}$ & $\begin{array}{c}A_{L}^{3,7} \\
0.124\end{array}$ & $\begin{array}{l}A_{L}^{4,7} \\
0.12\end{array}$ & $\begin{array}{c}A_{L}^{5,7} \\
0.122\end{array}$ & & & & & \\
\hline Model 3 & $S_{|t|}^{l, 1}$ & $S_{|t|}^{l, 2}$ & $S_{|t|}^{l, 3}$ & $S_{|t|}^{l, 4}$ & $S_{|t|}^{l, 5}$ & $S_{|t|}^{l, 6}$ & $S_{|t|}^{l, 7}$ & $S_{|t|}^{l, 8}$ & $S_{|t|}^{l, 9}$ & $S_{|t|}^{l, 10}$ & $S_{|t|}^{l, 11}$ & $S_{|t|}^{l, 12}$ & $S_{|t|}^{l, 13}$ \\
\hline$l=1$ & 0.452 & 0.662 & 0.454 & 0.406 & 0.466 & 0.694 & 0.702 & 0.814 & 0.788 & 0.57 & 0.41 & 0.254 & 0.296 \\
\hline$l=2$ & 0.428 & 0.552 & 0.218 & 0.464 & 0.436 & 0.678 & 0.652 & 0.816 & 0.684 & 0.55 & 0.41 & 0.276 & 0.296 \\
\hline$l=3$ & 0.476 & 0.784 & 0.454 & 0.424 & 0.466 & 0.66 & 0.602 & 0.874 & 0.782 & 0.582 & 0.43 & 0.206 & 0.296 \\
\hline$l=4$ & 0.656 & 0.738 & 0.676 & 0.55 & 0.584 & 0.72 & 0.807 & 0.884 & 0.82 & 0.584 & 0.386 & 0.224 & 0.296 \\
\hline \multirow[t]{2}{*}{$l=5$} & 0.652 & 0.538 & 0.422 & 0.444 & 0.536 & 0.78 & 0.836 & 0.844 & 0.842 & 0.578 & 0.416 & 0.244 & 0.296 \\
\hline & $A_{|t|}^{l, 1}$ & $A_{|t|}^{l, 2}$ & $A_{|t|}^{l, 3}$ & $A_{|t|}^{l, 4}$ & $A_{|t|}^{l, 5}$ & $A_{|t|}^{l, 6}$ & $A_{|t|}^{l, 7}$ & $A_{|t|}^{l, 8}$ & $A_{|t|}^{l, 9}$ & $A_{|t|}^{l, 10}$ & $A_{|t|}^{l, 11}$ & $A_{|t|}^{l, 12}$ & $A_{|t|}^{l, 13}$ \\
\hline$l=1$ & 0.442 & 0.644 & 0.43 & 0.39 & 0.568 & 0.682 & 0.856 & 0.822 & 0.818 & 0.596 & 0.45 & 0.278 & 0.294 \\
\hline$l=2$ & 0.402 & 0.446 & 0.318 & 0.362 & 0.326 & 0.488 & 0.674 & 0.834 & 0.72 & 0.57 & 0.446 & 0.308 & 0.296 \\
\hline$l=3$ & 0.606 & 0.748 & 0.446 & 0.388 & 0.454 & 0.558 & 0.804 & 0.868 & 0.79 & 0.606 & 0.434 & 0.23 & 0.294 \\
\hline$l=4$ & 0.638 & 0.768 & 0.666 & 0.348 & 0.58 & 0.722 & 0.86 & 0.874 & 0.824 & 0.584 & 0.4 & 0.226 & 0.296 \\
\hline \multirow[t]{2}{*}{$l=5$} & 0.632 & 0.514 & 0.404 & 0.432 & 0.522 & 0.722 & 0.83 & 0.852 & 0.86 & 0.582 & 0.412 & 0.246 & 0.39 \\
\hline & $S_{C}^{l, 13}$ & $A_{C}^{l, 13}$ & & & & & & & & & & & \\
\hline$l=1$ & 0.614 & 0.596 & & & & & & & & & & & \\
\hline$l=2$ & 0.48 & 0.454 & & & & & & & & & & & \\
\hline$l=3$ & 0.494 & 0.482 & & & & & & & & & & & \\
\hline$l=4$ & 0.51 & 0.478 & & & & & & & & & & & \\
\hline$l=5$ & 0.488 & 0.472 & & & & & & & & & & & \\
\hline$C=7$ & $\begin{array}{c}S_{L}^{2,7} \\
0.518\end{array}$ & $\begin{array}{c}S_{L}^{3,7} \\
0.566\end{array}$ & $\begin{array}{c}S_{L}^{4,7} \\
0.548\end{array}$ & $\begin{array}{c}S_{L}^{5,7} \\
0.598\end{array}$ & $\begin{array}{c}A_{L}^{2,7} \\
0.534\end{array}$ & $\begin{array}{c}A_{L}^{3,7} \\
0.578\end{array}$ & $\begin{array}{c}A_{L}^{4,7} \\
0.564\end{array}$ & $\begin{array}{c}A_{L}^{5,7} \\
0.618\end{array}$ & & & & & \\
\hline
\end{tabular}

Notes: 13 autocontours $C=[0.01,0.05,0.1,0.2,0.3,0.4,0.5,0.6,0.7,0.8,0.9,0.95,0.99]$.

$S_{|t|}^{l, 7}, A_{|t|}^{l, 7}$ for $l=1,2, \ldots 5 ; 7$ refers to the $50 \%$ autocontour.

$S_{L}^{l, 7}, A_{L}^{l, 7}$ stacking lags up to $l=2, \ldots .5$ and considering the $50 \%$ autocontour.

$S_{C}^{l, 13}$ and $A_{C}^{l, 13}$ stacking all 13 autocontours for one lag $l=1,2,3,4,5$.

500 bootstrap samples.

$\mathrm{T}=649, \mathrm{R}=360, \mathrm{P}=289, m=0.69$

Table 11: Bootstrapped P-values: Non-Linear Phillips Curve (Fixed Scheme) 